\title{
Gravitational phenomenology in higher-dimensional theories and strings
}

\author{
V. Alan Kostelecký and Stuart Samuel* \\ Physics Department, Indiana University, Bloomington, Indiana 47405
}

(Received 6 March 1989)

\begin{abstract}
We investigate gravitational phenomenology in compactified higher-dimensional theories, with particular emphasis on the consequences in string theory of tensor-induced spontaneous Lorentzsymmetry breaking. The role played by this mechanism in causing a gravitational version of the Higgs effect and in compactification is explored. The experimental viability of compactified theories with zero modes is considered by examining nonleading but observable gravitational effects. Additional constraints from the observed cosmological properties of the Universe are uncovered. Our investigations significantly constrain many theories involving extra dimensions in their perturbative regime. To resolve the phenomenological difficulties one must generate masses for the higherdimensional components of the metric while leaving massless the physical spacetime components. Some possibilities for overcoming this metric-mass problem are suggested. An open issue is whether the metric-mass problem is resolved in string theory.
\end{abstract}

\section{INTRODUCTION}

One intriguing notion in theoretical physics is the possibility that the Universe has more than four dimensions. From its origins ${ }^{1,2}$ early this century, this idea has grown to play an essential part in modern approaches to unification of gravity and the fundamental forces, such as Kaluza-Klein, ${ }^{3}$ supergravity, ${ }^{4}$ or string ${ }^{5}$ theories.

To establish the physical content of a typical $D$ dimensional theory, one usually assumes the higherdimensional spacetime manifold factors into two pieces, one of which is four-dimensional: $M^{D} \simeq M^{4} \times M^{n}$, where $n=D-4$ is the number of compactified dimensions. The four-dimensional interpretation of higher-dimensional fields is then found by Fourier expansion in the additional dimensions. This procedure requires the breaking of the invariance group of transformations of $M^{D}$, which normally is the $D$-dimensional Poincaré group. The breaking is implicitly assumed to occur via some unspecified mechanism.

Recently, a natural mechanism for spontaneous Lorentz-symmetry breaking has been found for the open-bosonic-string theory. ${ }^{6}$ It is intrinsic to strings in that it cannot occur in renormalizable particle theories.

The basic idea is as follows. Open-string field theory ${ }^{7}$ contains cubic interaction terms of the form $S T^{M} T_{M}$, where $S$ is a generic Lorentz-scalar field and $T^{M}$ is a generic Lorentz-tensor field with $M$ representing one or more Lorentz-vector indices. If one or more scalars $S$ have finite vacuum expectation values of the appropriate sign and magnitude, some Lorentz tensors $T^{M}$ acquire masssquared terms of the wrong sign. These tensor fields then also acquire expectation values, resulting in spontaneous breaking of the Lorentz group. In the bosonic string, a candidate scalar is the tachyon because it has a negative square mass in the naive perturbative vacuum. This signals a vacuum instability and therefore may even be desirable, given the phenomenological need to break the $D$-dimensional Poincaré symmetry and the large gauge groups often present in strings.
This breaking is called tensor-induced spontaneous Lorentz-symmetry breaking or tensor-induced breaking. It should be a possibility in many string theories. For example, in the closed bosonic string a tachyon is present and any field-theory formulation must contain trilinear couplings of the form $S T^{M} T_{M}$ because these couplings exist on shell. In string theory, such couplings can coexist with gauge symmetries because strings contain an infinite number of particle fields. In contrast, the mechanism cannot be implemented in renormalizable particle field theories with gauge symmetry. This is because gauge theories have scalar-tensor cubic couplings of the form $A^{\mu} \phi \partial_{\mu} \phi$ or $A^{\mu} A_{\mu} \phi \phi$ that either involve derivatives or generate positive masses for the vector $A_{\mu}$.

Thus, strings contain an attractive mechanism for breaking the higher-dimensional Poincaré symmetry. It is then natural to ask whether the mechanism is associated with the compactification of the extra dimensions. One goal of this paper is to address this question.

Intuition suggests that gravity should play a key role. However, gravity is a feature of closed-string theories for which an explicit field theory with calculable couplings has yet to be formulated. This means that the direct analysis of the role of tensor-induced breaking in compactification is impractical at present.

Instead, we confront the problem via another route. In the absence of an explicit realization of the breaking mechanism in a closed-string field theory, insight can be gained by constructing and analyzing via standard methods a $D$-dimensional effective field theory containing the essential features of the string case. The model action considered in this paper is the Einstein-Maxwell system with a potential for the vector field that induces spontaneous Lorentz-symmetry breaking. For this model we investigate the consequences of tensor-induced breaking on various features of the theory, including the resulting four-dimensional gravity. We explore the stability of uncompactified higher dimensions, the existence of a Higgs effect for gravity, and constraints on the structure of the internal manifold.

In addition to the need to reproduce precisely four 
macroscopic dimensions, higher-dimensional theories must satisfy other more subtle constraints. In particular, measurable gravitational effects can be induced by the presence of extra dimensions, ${ }^{8}$ even when the latter cannot directly be detected. The nonobservation of these effects places restrictions on the viability of many higher-dimensional theories, including strings. Another goal of this paper is the detailed exploration of these issues. We obtain solutions to the higher-dimensional equations of motion in the presence of a localized matter distribution, we examine their compatibility with experimental observation, and we seek possible phenomenological implications of tensor-induced spontaneous Lorentzsymmetry breaking.

Further constraints on compactified higher-dimensional theories arise from the experimental observation of the large-scale structure of the Universe. Here, we use the perfect-fluid approximation to determine the time evolution of cosmological models. The predictions of these are then compared with current experimental data. Since such models typically involve symmetric spaces, which are excluded when Lorentz symmetry is broken, we treat this topic in a pure Einstein theory.

The upshot of our analysis is that model builders should address two problems. The first is the problem of damping the propagation of massless higher-dimensional components of the metric: these are scalars with respect to the four-dimensional Lorentz group and induce undesirable phenomenological gravitational effects. The second is the asymmetry problem: one must ensure that the mechanism resolving the first problem is inoperative in the four physical dimensions. In the last part of this paper we mention several possible solutions to the first problem. The structure of string theories may be sufficiently complex to allow for one of these possibilities.

The organization of this paper is as follows. Section II establishes the string-motivated model Lagrangian with tensor-induced spontaneous Lorentz-symmetry breaking. Also, the equations determining the physics of the system are derived. Discussions of the possibility of a Higgs mechanism for gravity, the stability of uncompactified $D$-dimensional spacetime, and topological constraints on the internal manifold are presented in Sec. III. In Sec. IV the solution of the equations of motion in the presence of a localized matter distribution is obtained. The phenomenological consequences are discussed. Section V treats the cosmology of higherdimensional models and the associated phenomenology. We discuss the results and conclude in Sec. VI. Appendix A contains a proof of a topological result used in Sec. III, while some details concerning the form used in Sec. $\mathrm{V}$ for the stress-energy tensor for the internal space are relegated to Appendix $B$. We follow the conventions of Misner, Thorne, and Wheeler ${ }^{9}$ with the exception that in Sec. V the speed of light $c$ is sometimes explicitly displayed.

\section{A STRING-INSPIRED MODEL}

A string-inspired model for tensor-induced spontaneous Lorentz-symmetry breaking is established in this sec- tion. We find a background solution and obtain the equations determining small fluctuations about it. Properties of the model are explored in Sec. III.

As outlined in the Introduction, the idea is to find a model containing the essential features of the effective action that would arise in a string theory with tensorinduced breaking. This breaking is signaled in the Lagrangian by a mass-squared term of the wrong sign for some Lorentz tensor fields. Let us consider the simplest model, for which the only tensor field is a vector $A_{\mu}$. This situation most closely resembles the open bosonic string, ${ }^{6}$ with $A_{\mu}$ identified as the massless vector field. An effective action for $A_{\mu}$ and the metric $g_{\mu \nu}$ can be constructed by integrating over all other fields in the string theory.

Neglecting higher-derivative effects, this action is described ${ }^{10}$ by the Einstein-Maxwell action in $D$ dimensions with a potential $V=V\left(A_{\mu} A^{\mu}-a^{2}\right)$ for $A_{\mu}$ causing the spontaneous breaking of Lorentz symmetry:

$I=\int d^{D} x \sqrt{-g}\left[\frac{1}{16 \pi G_{D}} R-\frac{1}{4} F_{\mu v} F^{\mu v}-\frac{1}{2} V\right]$.

In this equation, $G_{D}$ is the $D$-dimensional Newton coupling constant, $F_{\mu \nu}=\partial_{\mu} A_{\nu}-\partial_{v} A_{\mu}$, and indices are raised using $g^{\mu \nu}$. The parameter $a$ in $V$ is a constant and $V(x)$ is positive except at $x=0$, where it vanishes.

Explicit forms for $V$ help to gain insight. One possibility is $V_{1}=(\lambda / 2)\left(A_{\mu} A^{\mu}-a^{2}\right)$, where $\lambda$ is a Lagrangemultiplier field. This useful choice effectively freezes motion about the potential minimum and hence permits an efficient extraction of the essential physics. It corresponds to a nonlinear $\sigma$ model. Another choice of $V$ pertinent to the analyses of small fluctuations and hence of the mass structure of the theory is $V_{2}=(\lambda / 2)\left(A_{\mu} A^{\mu}-a^{2}\right)^{2}$, where now $\lambda$ is a coupling constant.

The stress-energy tensor $T_{\mu v}$ is

$$
T_{\mu \nu}=-\frac{1}{4} g_{\mu \nu} F_{\rho \sigma} F^{\rho \sigma}+F_{\mu \gamma} F_{v}^{\gamma}-g_{\mu \nu} V+2 V^{\prime} A_{\mu} A_{v},
$$

where

$$
V^{\prime}=\left.\frac{\partial V(x)}{\partial x}\right|_{x=A_{\mu} A^{\mu}-a^{2}} .
$$

Using the Einstein tensor $G_{\mu \nu}=R_{\mu \nu}-\frac{1}{2} g_{\mu \nu} R$ and varying the action yields the equations of motion

$$
G_{\mu \nu}=8 \pi G_{D} T_{\mu \nu}, \quad F_{; \mu}^{\nu \mu}=-2 A^{v} V^{\prime} .
$$

For the potential $V_{1}$, one gets in addition to (2.4) the equation

$$
A^{\mu} A_{\mu}=a^{2},
$$

from varying $\lambda$.

A solution of Eq. (2.4) is

$$
g_{\mu v}=\eta_{\mu \nu}, \quad A_{\mu}=a_{\mu}, \quad \lambda=0 \text { if } V=V_{1},
$$

where $a_{\mu}$ is a constant vector satisfying $a_{\mu} a^{\mu}=a^{2}$. Perturbing Eqs. (2.4) and (2.5) about this vacuum solution, 


$$
\begin{aligned}
& g_{\mu \nu}=\eta_{\mu \nu}+h_{\mu \nu}, \quad\left|h_{\mu \nu}\right| \ll 1, \\
& A_{\mu}=a_{\mu}+\epsilon_{\mu}, \quad\left|\epsilon_{\mu}\right| \ll\left|a_{\mu}\right|, \\
& \lambda=0+\lambda_{1} \text { if } V=V_{1},
\end{aligned}
$$

yields the linearized equations for small fluctuations.

For the case $V=V_{1}$, these equations are

$$
\begin{aligned}
\partial_{\rho} \partial^{\rho} h_{\mu \nu}-\eta_{\mu \nu} \partial_{\rho} \partial^{\rho} h & +\eta_{\mu \nu} \partial^{\alpha} \partial^{\beta} h_{\alpha \beta}-\partial_{\mu} \partial^{\alpha} h_{v \alpha} \\
& -\partial_{v} \partial^{\alpha} h_{\mu \alpha}+\partial_{\mu} \partial_{v} h=-16 \pi G_{D} \lambda_{1} a_{\mu} a_{v},
\end{aligned}
$$

$\partial^{\mu} f_{\mu \nu}=\lambda_{1} a_{v}, \quad 2 \epsilon_{\mu} a^{\mu}=h^{\mu v} a_{\mu} a_{v}$,

where $f_{\mu \nu}=\partial_{\mu} \epsilon_{v}-\partial_{\nu} \epsilon_{\mu}$. The Bianchi identities imply

$$
\partial^{v}\left(a_{v} \lambda_{1}\right)=0 \text {. }
$$

Without loss of generality we can assume the background field $a_{\mu}$ is aligned in the $d$ th direction, where $d$ is one of the $D$ possibilities. Thus,

$$
a_{\mu}=a \eta_{\mu d}
$$

Eliminating $\lambda_{1}$ from Eq. (2.8) then yields

$$
\begin{aligned}
\partial_{\rho} \partial^{\rho} h_{\mu \nu}-\eta_{\mu \nu} \partial_{\rho} \partial^{\rho} h & +\eta_{\mu \nu} \partial^{\alpha} \partial^{\beta} h_{\alpha \beta}-\partial_{\mu} \partial^{\alpha} h_{v \alpha} \\
& -\partial_{\nu} \partial^{\alpha} h_{\mu \alpha}+\partial_{\mu} \partial_{v} h=-\frac{2 k}{a} \eta_{\mu d} \partial^{\alpha} f_{\alpha \nu},
\end{aligned}
$$

$2 \epsilon_{d}=a h_{d d}$,

where

$$
k=8 \pi G_{D} a^{2} .
$$

For potentials $V$ other than $V_{1}$, the equations governing the small fluctuations are

$$
\begin{aligned}
& \partial_{\rho} \partial^{\rho} h_{\mu \nu}-\eta_{\mu \nu} \partial_{\rho} \partial^{\rho} h+\eta_{\mu \nu} \partial^{\alpha} \partial^{\beta} h_{\alpha \beta}-\partial_{\mu} \partial^{\alpha} h_{v \alpha}-\partial_{\nu} \partial^{\alpha} h_{\mu \alpha}+\partial_{\mu} \partial_{v} h=-32 \pi G_{D} V^{\prime \prime} a_{\mu} a_{v}\left(2 \epsilon_{\alpha} a^{\alpha}-a_{\alpha} h^{\alpha \beta} a_{\beta}\right), \\
& \partial^{\mu} f_{\mu \nu}=2 V^{\prime \prime} a_{v}\left(2 \epsilon_{\alpha} a^{\alpha}-a_{\alpha} h^{\alpha \beta} a_{\beta}\right),
\end{aligned}
$$

where $V^{\prime \prime}$ is defined in a manner similar to $V^{\prime}$ in Eq. (2.3).

The linearized equations (2.8) or (2.13) are invariant under the infinitesimal local transformations

$$
\delta h_{\mu v}=\partial_{\mu} \chi_{v}+\partial_{\nu} \chi_{\mu}, \quad \delta \epsilon_{\mu}=\partial_{\mu}\left(\chi_{\nu} a^{v}\right),
$$

where $\partial_{\mu} a^{\nu}=0$. For the nonlinear $\sigma$ model, these are supplemented by $\delta \lambda_{1}=0$. Equation (2.14) is the original invariance of the theory under general coordinate transformations. It should not be confused with a $U(1)$ gauge transformation, precluded here by the potential $V$ for $A_{\mu}$.

An acceptable gauge choice for $h_{\mu \nu}$ is given by the functional constraint $f\left[h_{\mu \nu}\right]=0$, if there is a solution for $\chi_{\mu}$ to the equation $f\left[h_{\mu \nu}+\delta h_{\mu \nu}\right]=f\left[h_{\mu \nu}\right]+f\left[\delta h_{\mu \nu}\right]=0$ with $\delta h_{\mu v}$ given in terms of $\chi$ by Eq. (2.14). A useful gauge choice is the restriction to harmonic coordinates, $\partial^{\mu} \bar{h}_{\mu \nu}=0$, where $\bar{h}_{\mu \nu}=h_{\mu \nu}-\frac{1}{2} \eta_{\mu \nu} h$.

If the potential $V$ is other than $V_{1}$ and satisfies $V^{\prime \prime}(0)=\lambda \neq 0$, there is a massive mode $M$ corresponding to fluctuations about the potential minimum and given by the linear combination $2 \epsilon_{\alpha} a^{\alpha}-a_{\alpha} h^{\alpha \beta} a_{\beta}$. Its mass is

$$
m_{M}^{2}=4 \lambda a^{2}\left[1+\left(\frac{n+1}{n+2}\right) k\right],
$$

where $n=D-4$

\section{PROPERTIES OF THE MODEL}

This section discusses some general properties of the string-inspired model presented in Sec. III. We consider the question of the stability of the model, the possibility of a gravitational Higgs-type mechanism, and topological restrictions on the compactification.

\section{A. Stability of uncompactified higher dimensions}

One natural question to address is whether tensorinduced spontaneous Lorentz-symmetry breaking destabilizes a given background metric. An instability might be an indication that compactification of the extra dimensions is favored. To investigate this question let us examine the second-order fluctuations of the action. Denoting by $g_{\mu \nu}$ and $A_{\mu}$ the background fields and $h_{\mu \nu}$ and $\epsilon_{\mu}$ the associated fluctuations, the general second-order variation of the action (2.1) is

$$
\left.\delta^{2} I\right|_{\mathrm{eq} \mathrm{mot}}=\int d^{D} x \sqrt{-g}\left(\mathcal{L}_{h h}+\mathcal{L}_{h \epsilon}+\mathcal{L}_{\epsilon \epsilon}\right),
$$

where

$$
\begin{aligned}
\mathcal{L}_{h h}= & -\frac{h^{\mu \nu}}{32 \pi G_{D}}\left[g_{\mu \nu}\left(h_{; \rho}{ }^{\rho}-h_{; \rho \beta}^{\rho \beta}+h^{\beta \rho} R_{\beta \rho}\right)-h_{\mu \nu} R+\left(h^{\rho}{ }_{v ; \mu \rho}+h^{\rho}{ }_{\mu ; \nu \rho}-h_{\mu \nu ; \rho}{ }^{\rho}-h_{; \mu \nu}\right)\right] \\
& +\frac{1}{2} h^{\mu \nu}\left\{-h_{\mu \nu}\left(\frac{1}{4} F_{\alpha \beta} F^{\alpha \beta}+V\right)+h^{\gamma \delta}\left[-F_{\mu \gamma} F_{\nu \delta}+\frac{1}{2} g_{\mu \nu} F_{\gamma \sigma} F_{\delta}{ }^{\sigma}+A_{\gamma} A_{\delta}\left(g_{\mu \nu} V^{\prime}-2 A_{\mu} A_{v} V^{\prime \prime}\right)\right]\right\} \\
\mathcal{L}_{h \epsilon}= & h^{\mu \nu}\left[-\frac{1}{2} g_{\mu \nu} f_{\alpha \beta} F^{\alpha \beta}+2 f_{\mu \gamma} F_{v}^{\gamma}+2 \epsilon_{\gamma} A^{\gamma}\left(2 V^{\prime \prime} A_{\mu} A_{v}-g_{\mu \nu} V^{\prime}\right)+4 \epsilon_{\mu} A_{v} V^{\prime}\right], \\
\mathcal{L}_{\epsilon \epsilon}= & -\frac{1}{2} f_{\mu \nu} f^{\mu \nu}-2 \epsilon_{\mu} \epsilon^{\mu} V^{\prime}-4 \epsilon_{\mu} A^{\mu} \epsilon_{v} A^{v} V^{\prime \prime} .
\end{aligned}
$$

In these equations $R, F_{\mu \nu}, V, V^{\prime}$, and $V^{\prime \prime}$ are functions of the background fields, while $f_{\mu \nu}=\partial_{\mu} \epsilon_{v}-\partial_{\nu} \epsilon_{\mu}$ is the field strength for the fluctuation $\epsilon_{\mu}$. Upper indices are raised with the inverse background metric $g^{\mu \nu}$. We have freely used the equations of motion (2.4) to simplify the expressions in (3.2).

With our conventions, ${ }^{9}$ the background solution is destabilized by the vacuum expectation value of $A_{\mu}$ when the 
quadratic form in (3.1) has a positive eigenvalue. Whether this is the case for a generic background solution is a difficult question because of the intricate nature of (3.2). Let us analyze the case for a flat background. We set $g_{\mu \nu}=\eta_{\mu \nu}$ and $A_{\mu}=a_{\mu}=a \delta_{\mu d}$. The second-order variation (3.1) becomes

$$
\delta^{2} I \approx \int d^{D} x\left[\frac{1}{32 \pi G_{D}}\left(h^{\mu v} \partial_{\rho} \partial^{\rho} h_{\mu \nu}-h \partial_{\rho} \partial^{\rho} h+2 h \partial^{\mu} \partial^{v} h_{\mu v}+2 \partial_{\lambda} h^{\lambda \mu} \partial^{v} h_{\mu v}\right)-\frac{1}{2} f^{\mu v} f_{\mu \nu}-\left(a^{\mu} h_{\mu \nu} a^{v}-2 \epsilon_{\mu} a^{\mu}\right)^{2} V^{\prime \prime}(0)\right]
$$

The first line in (3.3) contains the flat-space background fluctuations for ordinary gravity. They are stable after a gravitational gauge is fixed. The remaining two terms in (3.3) are negatives of perfect squares and hence are negative definite. This means that $D$-dimensional spacetime is stable to perturbations.

\section{B. Absence of a gravitational Higgs mechanism}

In particle theories, an explicit mass term in the Lagrangian for any gauge field is forbidden by gauge invariance. Spontaneous symmetry breaking bypasses this problem, allowing mass generation via the Higgs effect. Non-Abelian gauge bosons $A_{\mu}^{j}$ couple to a Higgs field $\phi_{a}$ via a covariant derivative $D_{\mu} \phi_{a}=\partial_{\mu} \phi_{a}-A_{\mu}^{j} L_{a b}^{j} \phi_{b}$, where $L_{a b}^{j}$ is the $j$ th generator for the gauge group and $a$ and $b$ are matrix indices. The kinetic term for $\phi$ involves $D^{\mu} \phi_{a} D_{\mu} \phi_{a}$ and hence the term $A_{\mu}^{i} L_{a b}^{i} \phi_{b} A^{\mu j} L_{a c}^{j} \phi_{c}$. When $\phi_{a}$ acquires a vacuum expectation value, this term generates a mass matrix for $A_{\mu}^{j}$. This mechanism is fundamental in generating masses for the $W$ and $Z$ bosons in the electroweak model, for example.

Gravity may be viewed as analogous to a non-Abelian gauge theory with the connection and the Lorentz group playing the roles of the gauge field and the gauge group, respectively. ${ }^{11}$ As the Higgs mechanism plays such a basic role in particle theory, it is natural to investigate whether a gravitational version occurs for which certain components of $h_{\mu \nu}$ become massive. ${ }^{12}$

For our model, $A_{\mu}$ plays the role of the Higgs field $\phi_{a}$. The potential $V$ is minimized when $A_{\mu}$ acquires a constant expectation value $a \delta_{\mu D-1}$. In analogy to nonAbelian gauge theory we seek a longitudinal component of the metric arising from the nonzero expectation value of the vector field in the kinetic term. However, a nonzero expectation value for $A_{\mu}$ gives no new contribution to $h_{\mu v}$ in $F^{\mu v} F_{\mu \nu}$ because the affine connection coupling gravity to tensor fields cancels in the antisymmetric combination $F_{\mu \nu}=\partial_{\mu} A_{v}-\partial_{\nu} A_{\mu}$. The field $A_{\mu}$ does not become a Higgs field but remains a Goldstone boson.

Although the above argument appears specific to the use of $F^{\mu v} F_{\mu v}$ as a kinetic-energy term, the absence of a Higgs effect holds quite generally for spontaneous Lorentz-symmetry breaking induced by any tensor fields, for the following reason. The generators of general coordinate transformations involve spacetime derivatives. The gravitational analogue of $L_{a b}^{j}$ is an operator involving $\partial_{\mu}$. When a tensor field acquires an expectation value, instead of generating a quadratic form involving the square of $L_{a b}^{j}$, a quadratic form bilinear in $\partial_{\rho} h_{\mu v}$ results.
This may distort the propagation of gravitons but does not dampen their effects at large distances. Thus, no mass terms arise.

The argument given above is sufficiently general to apply when more than one tensor field acquires a nonzero expectation value. This means that metric-mass generation arising from tensor-induced breaking is unlikely even in a full string theory.

An alternative mechanism for metric-mass generation might proceed through the potential $V=V\left(A_{\mu} g^{\mu v} A_{v}\right.$ $-a^{2}$ ). Since this depends on $g_{\mu v}$ as well as on $A_{\mu}$, a quadratic derivative-free term for the metric might arise. The analogous situation does not occur in a non-Abelian gauge theory because the scalar potential does not contain the gauge field. If it occurs, this alternative mechanism would necessarily be qualitatively different from the usual Higgs mechanism in particle theory.

However, an analysis of the Lagrangian (3.2) for fluctuations reveals that the combination $2 \epsilon_{\mu} g^{\mu v} A_{v}$ $-A_{\mu} h^{\mu v} A_{v}$ is massive, rather than a component of $h_{\mu v}$. The orthogonal combination $\epsilon_{\mu} g^{\mu v} A_{v}+2 A_{\mu} h^{\mu v} A_{v}$ remains massless.

\section{Topological constraints on compactification}

Suppose that a solution for the gravitational sector can be found that has the form $M^{4} \times M^{n}$, where $M^{4}$ is a fourdimensional flat space and $M^{n}$ is a compact $n$ dimensional manifold. One solution for $A_{\mu}$ minimizing the terms

$$
\mathcal{L}_{g A}=\frac{1}{4} F^{\mu v} F_{\mu v}+\frac{1}{2} V
$$

in Eq. (2.1) is $A_{\mu}=a \delta_{\mu d}$, where $d$ points in one of the three physical dimensions. This implies Lorentzsymmetry breaking in the physical dimensions, which is undesirable. It is therefore necessary to have a competitive solution $A_{\mu}=a_{\mu}$ lying in $M^{n}$ and rendering zero each term in (3.4).

From these requirements, general topological features of the compactified manifold may be obtained. Viewing $a_{\mu}$ as the components of a one-form $\hat{a}=a_{\mu} d x^{\mu}$, the vanishing of the first term in Eq. (3.4) implies that $\hat{a}$ is closed; i.e., $d \hat{a}=0$. Viewing $a^{\mu}$ as the components of a vector field a, the vanishing of the second term in Eq. (3.4) implies that $\boldsymbol{M}^{n}$ possesses a nowhere-vanishing vector field of constant magnitude. This vector may be normalized to 1 by dividing $a^{\mu}$ by $a \equiv|\mathbf{a}|$. The condition $\mathbf{a} \cdot \mathbf{a}=1$ can also be written $\widehat{a}(\mathbf{a}) \equiv\langle\widehat{a}, \mathbf{a}\rangle=1$.

Summarizing, on $M^{n}$ there must exist (i) a vector field a with $\mathbf{a} \cdot \mathbf{a}=1$ and (ii) a closed nowhere-vanishing oneform $\hat{a}$. 
First, consider the consequences of condition (i) alone. A compact manifold $M^{n}$ has a nowhere-vanishing vector field if and only if its Euler characteristic $\chi\left(M^{n}\right)$ is zero. ${ }^{13}$ Examples of such spaces are the Lie-group manifolds and the odd-dimensional spheres $S^{2 n+1}$.

Next, consider condition (ii) alone. Recall that the first de Rham cohomology group $H^{1}\left(M^{n}, \mathbb{R}\right)$ comprises closed one-forms $\omega$ modulo exact forms $d \lambda$, where $\lambda$ is a function on $M^{n}$. The dimension of this group is the first Betti number $b_{1}$. However, on a compact manifold $M^{n}$ it is impossible to have a nowhere-vanishing $\hat{a}=d \lambda$ : since $\lambda$ is a continuous function from $M^{n}$ to $\mathbb{R}$, it attains a maximum at some point in $M^{n}$ at which $d \lambda$ vanishes. Condition (ii) therefore implies that $b_{1} \neq 0$, i.e., $H^{1}\left(M^{n}, \mathbb{R}\right)$ must be nontrivial.

One class of manifolds satisfying both conditions (i) and (ii) consists of the product manifolds $N^{n-1} \times S^{1}$ for arbitrary compact $N^{n-1}$. The components of the nowhere-vanishing vector a can be chosen as $(0,0,0, \ldots, 0, u)$ where there are $n-1$ zeros and where $u$ is the unit vector on the circle; i.e., the vector a lies in the tangent space of the $S^{1}$ factor. The corresponding closed one-form $\hat{a}$ is $d \theta$, where $\theta$ is the standard variable parametrizing the circle, $0 \leq \theta \leq 2 \pi$, and $\theta=0$ is identified with $\theta=2 \pi$.

In the search for other manifolds satisfying conditions (i) and (ii), it is natural to examine $n$-dimensional Liegroup manifolds. These satisfy condition (i) because they are parallelizable; i.e., they possess $n$ linearly independent nonvanishing vector fields. If $\hat{a}$ is a right-invariant oneform, a basis $\omega^{j}, j=1, \ldots, n$ for all right-invariant oneforms can be chosen such that $\omega^{1}=\hat{a}$. The Cartan structure equations $d \omega^{j}=f^{j k l} \omega^{k} \omega^{l}$ together with $d \omega^{1}=0$ then imply $f^{1 k l}=0$. The Lie algebra therefore has a $u(1)$ factor.

One manifold that is not a product but that satisfies both (i) and (ii) is the Klein bottle. Take $S^{1} \times[0,1]$ where $[0,1]$ is the unit interval. Identifying $(s, 0)$ with $(s, 1)$ for each $s \in S^{1}, 0 \leq s \leq 2 \pi$ generates the torus. Identifying instead $(s, 0)$ with $(h(s), 1)$, where $h(s)=2 \pi-s$, generates the Klein bottle. It may be viewed as a fiber bundle over $S^{1}$ with fiber $S^{1}$. It is the product of a circle with the unit interval modulo an equivalence relation: $S^{1} \times[0,1]$ / $[(s, 0) \sim(h(s), 1)]$.

These examples provide insight as to the type of manifold compatible with conditions (i) and (ii). The circle in the above examples can be obtained by constructing the integral curve for the vector field $\hat{a}$. In general, this integral curve may not close. For example, if the manifold is the flat torus $S^{1} \times S^{1}$ and the vector field is aligned at an irrational angle then the integral curve wraps densely around the torus without closing. However, in this case the vector field could be taken instead in the tangent space of either $S^{1}$. It turns out that this feature is generic: appropriate perturbations always isolate a circle.

In fact, there exists a theorem ${ }^{14}$ stating that any smooth arcwise-connected compact manifold $M^{n}$ satisfying conditions (i) and (ii) is a fiber bundle over $S^{1}$. This means that

$$
M^{n} \simeq \frac{N^{n-1} \times[0,1]}{(s, 0) \sim(h(s), 1)},
$$

where the fiber is an $(n-1)$-dimensional manifold $N^{n-1}$ and $h$, called the monodromy, is a differentiable automorphism of $N^{n-1}$. Conversely, if $f: M^{n} \rightarrow S^{1}$ is a fiber bundle, then $f^{*} d \theta$ is a nowhere-vanishing closed one-form and one can give $M^{n}$ a Riemannian metric such that there is a vector field $\mathbf{v}$ satisfying $f^{*} d \theta(\mathbf{v})=1$ and $\mathbf{v} \cdot \mathbf{v}=1$. In Appendix $A$, a proof of this theorem due to Davis is presented. Many of the key ideas behind the proof are illustrated by the examples above.

The bundle may be trivialized, i.e., $M^{n} \cong N^{n-1} \times S^{1}$, if $h$ is isotropic to the identity $\mathbf{1}$. This means there exists a homotopy family $h_{t}$ for $0 \leq t \leq 1$ depending smoothly on $t$ such that $h_{0}=\mathbf{1}$ and $h_{1}=h$ with each $h_{t}$ a diffeomorphism. The map

$$
N^{n-1} \times \frac{[0,1]}{0 \sim 1} \rightarrow \frac{N^{n-1} \times[0,1]}{(s, 0) \sim(h(s), 1)}
$$

given by $(n, t) \mapsto\left(h_{t}(n), t\right)$ establishes the diffeomorphism between the two spaces.

This trivialization can be illustrated for the group manifold $\mathrm{U}(n)$. The elements $\mathbf{I} e^{2 \pi i m / n}$ for $m=1, \ldots, n$, where $\mathbf{I}$ is the $n \times n$ unit matrix, are in both $\mathrm{SU}(n)$ and $\mathrm{U}(1)$. Elements $u \in \mathrm{U}(n)$ may be projected from $\mathrm{U}(n)$ to $\mathrm{SU}(n)$ by the mapping $u \mapsto u /(\operatorname{det} u)^{1 / n}$. Therefore, any $u \in \mathrm{U}(n)$ is specified by the pair $(s, t)$ via $u=s e^{2 \pi i t / n}$, where $s \in \mathrm{SU}(n)$ and $t \in[0,1]$. With this representation we can write

$$
\mathrm{U}(n)=\frac{\mathrm{SU}(n) \times[0,1]}{(s, 0) \sim(h(s), 1)},
$$

where $h(s)=s e^{-2 \pi i / n}$. The $u \in \mathrm{U}(n)$ are correctly identified at $t=0$ and $t=1$ because the pair $(s, 0)$ represents the element $s$ and the pair $(h(s), 1)$ represents the element $h(s) e^{2 \pi i / n}=s$. The homotopy family $h_{t}$ connecting $\mathbf{1}$ to $h$ is given by the diagonal matrix with $e^{(-2 \pi i t / n)}$ for the first $n-1$ entries and $e^{-2 \pi i(1-n) t / n}$ for the last entry. Thus, the group manifold $\mathrm{U}(n)$ is a trivial $S^{1}$ bundle.

Finally, (3.5) may be generalized slightly. If tensorinduced spontaneous Lorentz-symmetry breaking corresponds to having $k$ linearly independent closed nowherevanishing one-forms then $M^{n}$ is a fiber bundle over a $k$ dimensional torus. ${ }^{14}$ The proof in Appendix A may be generalized to cover this case.

It is likely that analogous topological constraints on the internal manifold arise in the general string case if tensor-induced breaking is present. Note that the constraint $\chi\left(M^{n}\right)=0$ is undesirable for Calabi-Yau compactifications since $\left|\chi\left(M^{n}\right)\right| / 2$ is the number of generations in the standard model. ${ }^{15}$

\section{GENERALIZED SCHWARZSCHILD SOLUTIONS}

In this section we consider the modifications to the gravitational field of a localized matter distribution that arise in a higher-dimensional theory with spontaneous Lorentz-symmetry breaking. This is of interest because there now exist high-precision tests of Einstein gravity, ${ }^{16}$ for example, from the Viking Lander experiments. We find that these experiments impose interesting constraints on the nature of the extra dimensions. The results are 
applicable not only to the string-inspired model discussed in the previous sections but more generally to theories involving higher dimensions, such as Kaluza-Klein models and $D>4$ supergravity theories. Theories without tensor-induced spontaneous Lorentz-symmetry breaking are obtained from the analysis by taking the limit as the parameter $k$ of (2.12) vanishes.

Suppose material is introduced into our model, so that we can consider the gravitational field of a localized static distribution of total mass $M$. This might represent a planetary body or the Sun, for example. We are interested in the behavior of the four-dimensional gravitational potential at large radial distances $r$, where perturbative methods are applicable. In this section we determine the first few terms of the expansion in $M G_{N} r^{-1}$ of $h_{\mu v}$.

For the leading large- $r$ behavior, the detailed nature of the localized mass distribution is unimportant and thus can be approximated by a $\delta$ function at the origin. We check this idea by considering two types of $\delta$-function distribution for the stress-energy tensor, which we take to have the form $T_{\mu \nu}=\hat{\rho} \eta_{\mu 0} \eta_{\nu 0}$. The first is pointlike in the physical dimensions and uniformly distributed in the higher dimensions:

$$
\hat{\rho}_{1}=\frac{M}{V_{n}} \delta^{3}(\mathbf{x})
$$

where $V_{n}$ is the volume of the compactified manifold. The second is pointlike in all $D-1$ dimensions:

$$
\hat{\rho}_{2}=M \delta^{D-1}(\mathrm{x}) \text {. }
$$

Consider Eq. (2.1) in the limit of the nonlinear $\sigma$ model, for which $A_{\mu}$ satisfies $A_{\mu} A^{\mu}=a^{2}$. This limit is appropriate for investigating the leading large- $r$ behavior, and thus the results presented below are independent of the choice of potential $V$.

Equations (2.7) generate the fluctuations about the background metric:

$$
\begin{array}{r}
\partial_{\rho} \partial^{\rho} \bar{h}_{\mu \nu}+\eta_{\mu \nu} \partial^{\alpha} \partial^{\beta} \bar{h}_{\alpha \beta}-\partial_{\mu} \partial^{\alpha} \bar{h}_{\alpha \nu}-\partial_{\nu} \partial^{\alpha} \bar{h}_{\alpha \mu}+k \delta_{\mu d} \delta_{v d}\left(\partial_{\rho} \partial^{\rho} \bar{h}_{d d}-\partial_{d}^{2} \bar{h}_{d d}-\frac{1}{D-2}\left(\partial_{\rho} \partial^{\rho}-\partial_{d}^{2}\right) \bar{h}-\frac{2}{a} \sum_{\mu \neq d} \partial_{d} \partial^{\mu} \epsilon_{\mu}\right) \\
=-16 \pi G_{D} \hat{\rho} \eta_{\mu 0} \eta_{\nu 0}
\end{array}
$$

Here, we define $\bar{h} \equiv \eta^{\mu \nu} \bar{h}_{\mu \nu}$, we take the background vector $a_{\mu}$ to point in the $d$ th direction, and $k$ is defined in Eq. (2.12).

We are interested in a static solution with $\epsilon_{\mu}=0$. For definitiveness we take $d=D-1$ so that $A_{\mu}=a \delta_{\mu D-1}$. Furthermore, we choose the harmonic gauge $\partial^{\alpha} \bar{h}_{\alpha \beta}=0$. Then, Eq. (4.3) becomes

$$
\begin{aligned}
& \nabla^{2} \bar{h}_{\mu \nu}=0 . \\
& \nabla^{2} \bar{h}_{00}=-16 \pi G_{D} \hat{\rho}, \\
& (1+k) \nabla^{2} \bar{h}_{d d}-k \partial_{d}^{2} \bar{h}_{d d}+\frac{k}{D-2}\left(\partial_{d}^{2}-\nabla^{2}\right) \bar{h}=0 .
\end{aligned}
$$

In the first of these equations, $\mu=\nu=0$ and $\mu=\nu=d$ are excluded.

The first equation in (4.4) implies $\bar{h}_{\alpha \beta}=0$ except for $\alpha=\beta=0$ and $\alpha=\beta=d$. The second equation yields $\bar{h}_{00}$. With these results the third equation determines $\bar{h}_{d d}$.

Before discussing the general solution let us present an argument that the leading large-r behavior of $\bar{h}_{00}$ is $r^{-1}$ independent of the mass distribution. We assume for simplicity that the internal space is an $n$-dimensional torus $T^{n}$ with $T^{1}$ circumferences denoted by $L_{j}, j=1, \ldots, n$. The volume of this space is $V_{n}=\Pi_{j} L_{j}$.

Consider first the distribution $\rho_{1}$ of Eq. (4.1). For this case, $\bar{h}_{00}(\mathbf{x})$ is independent of the coordinates of the internal space and the solution becomes the $D=4$ Schwarzschild one for an effective density $M / V_{n}$ :

$$
\bar{h}_{00}=\frac{4 M G_{D}}{V_{n} r} \text {, }
$$

where $r^{2}=x^{2}+y^{2}+z^{2}$.
Next, consider the distribution $\rho_{2}$ of Eq. (4.2). The periodicity of the tori $T^{1}$ in $T^{n}$ means that the method of images can be used to obtain the solution for $\bar{h}_{00}$ from the solution of the Einstein equations in $D$ dimensions. We find

$\bar{h}_{00}=\frac{16 \pi M G_{D}}{c_{D}} \sum_{m_{j}=-\infty}^{\infty}\left(r^{2}+\sum_{j=1}^{n} m_{j}^{2} L_{j}^{2}\right)^{-(n+1) / 2}$,

where $c_{d}$ is defined by

$$
\nabla_{D-1}^{2} \frac{1}{r^{D-3}}=-c_{D} \delta^{D-1}(\mathbf{x})
$$

It is given explicitly as

$$
c_{D}=(D-3) S^{D-2}=\frac{2(D-3) \pi^{(D-1) / 2}}{\Gamma((D-1) / 2)},
$$

where $S^{p}$ is the $p$-volume of the $p$-dimensional sphere.

As $r \rightarrow \infty$, the sum over $m_{j}$ in (4.6) may be replaced by an integral. The result coincides with (4.5) if

$\frac{1}{4 \pi r V_{n}}=\frac{1}{c_{D}} \int_{-\infty}^{\infty} d x_{1} \cdots d x_{n}\left(r^{2}+\sum_{j=1}^{n} L_{j}^{2} x_{j}^{2}\right)^{-(n+1) / 2}$.

By changing variables via $x_{j}=z_{j} r / L_{j}$, Eq. (4.9) is satisfied if

$$
\int_{-\infty}^{\infty} d z_{1} \cdots d z_{n}\left[1+\sum_{j} z_{j}^{2}\right]^{-(n+1) / 2}=\frac{c_{D}}{4 \pi} .
$$


This formula can be proven by induction or by going to spherical coordinates in the $z_{j}$. Although we have only explicitly treated the toroidal case, physical considerations imply Eq. (4.5) holds for large $r$ independent of the detailed structure of the compact internal manifold.

We next present a general solution to Eq. (4.4):

$$
\begin{aligned}
& h_{00}=\frac{4 M G_{D}(l-1)}{V_{n} l r}, \\
& h_{j j}=\frac{4 M G_{D}}{V_{n} l r}, \\
& h_{D-1 D-1}=\frac{4 M G_{D}}{V_{n} l(k+1) r},
\end{aligned}
$$

where

$$
l=(1+k)^{-1}[(n+2)+(n+1) k] .
$$

To compare with experiment, introduce the expansion

$$
\begin{aligned}
d s^{2}= & -\left[1-2 M G_{N} r^{-1}+2 \beta\left(M G_{N} r^{-1}\right)^{2}+\cdots\right] d t^{2} \\
& +\left(1+2 \gamma M G_{N} r^{-1}+\cdots\right)\left(d r^{2}+r^{2} d \Omega^{2}\right)
\end{aligned}
$$

of the metric generated by a static, spherically symmetric body of mass $M$. In this expression, the EddingtonRobertson parameters ${ }^{17,18} \beta$ and $\gamma$ have experimental values currently determined as ${ }^{19,20}$

$$
\beta=1.003 \pm 0.005, \quad \gamma=1.000 \pm 0.002 \text {. }
$$

The first $r^{-1}$ term in $h_{00}$ fixes the definition of $M G_{N}$ as

$$
G_{n} \equiv \frac{2(l-1) G_{D}}{l V_{n}}
$$

With this definition, the Newton gravitational potential in $D$ dimensions coincides with the standard fourdimensional case. The result is independent of the detailed nature of the internal manifold.

Einstein gravity involves measurable corrections to Newton gravity. These are represented in lowest order by the $\beta$ and $\gamma$ terms in (4.13). Using Eqs. (4.11), (4.12), (4.13), and (4.15), the value for $\gamma$ is found to be

$$
\gamma=\frac{1+k}{1+n+n k},
$$

where $n \geq 1$. We have verified that the result (4.16) is independent of the choice of gravitational gauge, i.e., of harmonic coordinates. Equation (4.16) is incompatible with the experimental result (4.14) unless either $D=4$ and $k=0$ or $D=5$ and $k \rightarrow \infty$. The latter possibility is unnatural in the context of string theory since the scale of $a$ is set by the Planck scale and hence a value of $k$ of order 1 is expected.

Thus, the assumptions made lead to the conclusion that higher dimensions are excluded by established experimental data. The analysis holds at the classical level. As such, it is independent of other difficulties with higher dimensions such as natural chirality generation ${ }^{21}$ and renormalizability or finiteness.

One way to avoid the phenomenological difficulty with higher dimensions is to have only short-range forces associated with the metric components $h_{j k}, j, k \geq 4$. This can be achieved if these components obtain a mass. Their propagation would then be damped at large distances, so that, for example, the observed value of $\gamma$ would be 1 . This possibility has been considered in Ref. 8 and is further discussed in Sec. VI.

So far, we have assumed that the background vector $a_{\mu}$ is aligned in one of the internal dimensions. It is interesting to contemplate the possibility that it is instead aligned in one of the physical spacetime dimensions, $a_{\mu}=a \eta_{\mu 1}$, say. Tensor-induced spontaneous Lorentzsymmetry breaking in the physical space is not what is sought and probably causes phenomenological difficulties. However, in leading order there are solutions that are quite different from the one given above but that incorporate standard Newton gravity.

Consider the theory in the limit of the nonlinear $\sigma$ model. We are interested in a ground state, i.e., a solution to the equations of motion that satisfies the Bianchi identities and that minimizes the potential. One simple choice is $\lambda_{1}=0$. This decouples the equations for $h_{\mu \nu}$ and $a_{\mu}$, leaving only the constraint relation between $\epsilon_{1}$ and $h_{11}$. The gravitational solution is therefore the usual Schwarzschild one,

$$
\bar{h}_{00}=\frac{4 G_{N} M}{r} \text {, }
$$

with the other components of $h_{\mu \nu}$ being zero.

It remains to obtain a solution to the Maxwell equations $\partial^{\mu} f_{\mu \nu}=0$ subject to the constraint $\epsilon_{1}=\frac{1}{2} a h_{11}$ $=a M G_{N} / r$. The simplest choice, which minimizes the action, is $f_{\mu v}=0$. The solution is

$$
\begin{aligned}
& \epsilon_{1}=\frac{a M G_{N}}{r}, \\
& \epsilon_{2}=\frac{a M G_{N}}{r} \frac{y}{x+r}+\partial_{y} \Lambda(y, z), \\
& \epsilon_{3}=\frac{a M G_{N}}{r} \frac{z}{x+r}+\partial_{z} \Lambda(y, z),
\end{aligned}
$$

where $\Lambda(y, z)$ is an arbitrary function. Here, the equation for $G_{N}$ in (4.15) is replaced by $G_{N}=G_{D} / V_{n}$.

The components $\epsilon_{2}$ and $\epsilon_{3}$ of the vector potential in (4.18) each have a singularity along $x<0$, when $y \rightarrow 0$ and $z \rightarrow 0$. The singularity can be moved elsewhere by a suitable choice of $\Lambda$. For example, the choice $\Lambda=-a M G_{N} \ln \left(y^{2}+z^{2}\right)$ results in expressions for $\epsilon_{2}$ and $\epsilon_{3}$ similar to those of Eq. (4.18) but with $x+r$ replaced with $x-r$. The singularity is then along $x>0$. It is unphysical since it satisfies $\oint_{c} \epsilon \cdot d l=0$ for any contour $c$ taken about the $x$ axis.

There appears to be no significance to the bad behavior of the potential $\epsilon_{\mu}$ near $y=0$ and $z=0$, unlike the singularity of a Dirac monopole, for example, for which there is a physical basis. Note that the tensor-induced spontaneous Lorentz-symmetry breaking manifests itself here only through nonleading terms, in contrast to the usual situation when a symmetry is spontaneously broken, for 
which the effects of the breaking are quite apparent in the ground state.

\section{COSMOLOGY IN HIGHER DIMENSIONS}

In Sec. IV we found phenomenological constraints on higher-dimensional theories due to measurable nonleading gravitational effects of a mass distribution. It is natural to ask whether additional constraints arise from cosmological considerations. In general, we find that certain models are excluded but that the restrictions on higher-dimensional theories are less severe than those of Sec. IV. This is largely due to a lack of precise observational data.

It is natural and standard to investigate higherdimensional cosmologies by letting both the physical spatial dimensions and the compactified dimensions evolve separately as homogeneous maximally symmetric spaces. ${ }^{22-35}$ This assumption is incompatible with the mechanism for tensor-induced spontaneous Lorentzsymmetry breaking discussed in the previous sections. Consequently, we disregard here the effects of nonzero expectation values of tensor fields.

Throughout this section a subscript 0 on a quantity indicates its present-day value. For certain equations where comparison with experimental values is useful, the dependence on the speed of light $c$ is explicitly shown.

\section{A. The cosmological equations for arbitrary dimensions}

We consider cosmologies in a pure $D$-dimensional Einstein theory that involve two homogeneous spaces: a four-dimensional one and an $n$-dimensional one, governed by scale parameters $a(t)$ and $b(t)$, respectively. The metric is

$$
d s^{2}=-d t^{2}+a^{2}(t) g_{a b} d x^{a} d x^{b}+b^{2}(t) g_{j k} d x^{j} d x^{k},
$$

where $a=0, \ldots, 3$ labels the physical spacetime dimensions and $j=1, \ldots, n$ labels the internal dimensions.

We approximate the cosmological matter distribution as a perfect fluid with $D$-dimensional density $\hat{\rho}$, pressure $\hat{p}$ in the physical dimensions, and pressure $\hat{\tau}$ in the internal dimensions. The stress-energy tensor $T_{\mu \nu}$ is then given by the diagonal $D \times D$ matrix with entries $(\hat{\rho}, \hat{p}, \hat{p}, \hat{p}, \hat{\tau}, \ldots, \hat{\tau})$. Note that the four-dimensional density and pressure are given by $\rho=\hat{\rho} V_{n}$ and $p=\hat{p} V_{n}$. For the physical situation, where $b<<a$, a good approximation is $\hat{\tau}=0$, as is shown in Appendix B.

The Einstein equations $R_{\mu \nu}-\frac{1}{2} g_{\mu \nu}+\Lambda g_{\mu \nu}=8 \pi G_{D} T_{\mu \nu}$ for the case $n>0$ reduce to three coupled second-order differential equations for $a$ and $b$, with coefficients dependent on $D$ :

$$
\begin{aligned}
& {\left[\frac{\dot{a}}{a}\right]^{2}+n \frac{\dot{a}}{a} \frac{\dot{b}}{b}+\frac{n(n-1)}{6}\left[\frac{\dot{b}}{b}\right]^{2}=-\frac{k}{a^{2}}-\frac{n(n-1) k_{n}}{6 b^{2}}+\frac{\Lambda}{3}+\frac{8 \pi G_{D}}{3} \hat{\rho},} \\
& 2 \frac{\ddot{a}}{2}+\left[\frac{\dot{a}}{a}\right]^{2}+2 n \frac{\dot{a}}{a} \frac{\dot{b}}{b}+n \frac{\ddot{b}}{b}+\frac{n(n-1)}{2}\left[\frac{\dot{b}}{b}\right]^{2}=-\frac{k}{a^{2}}-\frac{n(n-1) k_{n}}{2 b^{2}}+\Lambda-8 \pi G_{D} \hat{p}, \\
& \frac{\ddot{a}}{a}+\left[\frac{\dot{a}}{a}\right]^{2}+(n-1) \frac{\dot{a}}{a} \frac{\dot{b}}{b}+\frac{(n-1)}{3} \frac{\dot{b}}{b}+\frac{(n-1)(n-2)}{6}\left[\frac{\dot{b}}{b}\right]^{2}=-\frac{k}{a^{2}}-\frac{(n-1)(n-2) k_{n}}{6 b^{2}}+\frac{\Lambda}{3}-\frac{8 \pi G_{D}}{3} \hat{\tau},
\end{aligned}
$$

where $k=-1,0$, or +1 determines whether the geometry of the four-dimensional space is open, flat, or closed. Likewise, $k_{n}$ determines the geometry of the $n$ dimensional space. For a compact internal space $k_{n}=1$ or 0 . The latter value occurs for toroidal compactifications, for example. The Bianchi identities follow from energy conservation and are

$$
\partial_{t}\left(\hat{\rho} a^{3} b^{n}\right)=-\hat{p} b^{n} \partial_{t} a^{3}-\hat{\tau} a^{3} \partial_{t} b^{n}
$$

This equation makes one of the three equations in (5.2) redundant.

The possibility that $k_{n}=1$ can be excluded for realistic evolutions, provided no special choice of the density $\hat{\rho}$ is made. We show a contradiction follows from the assumption that the present value $b_{0}$ of $b$ is microscopic.

Evaluate the first equation in (5.2) at the present time. Since $b_{0}$ is small, the $b^{-2}$ term dominates the right-hand side. Special choices of $\hat{\rho}$ can invalidate this but appear ad hoc and unnatural in the present context. Setting $r_{0}=\dot{b}_{0} / b_{0}$, one finds

$$
H_{0}^{2}+n H_{0} r_{0}+\frac{n(n-1)}{6} r_{0}^{2}+\frac{n(n-1)}{6 b_{0}^{2}}=0 \text {, }
$$

where $H_{0}$ is the Hubble constant $H_{0}=\dot{a}_{0} / a_{0}$. Equation (5.4) is a quadratic equation for $r_{0}$, which has real solutions only if the discriminant

$$
n^{2} H_{0}^{2}-2 \frac{n(n-1)}{3}\left(H_{0}^{2}+\frac{c^{2} n(n-1)}{6 b_{0}^{2}}\right)
$$

is positive. This occurs when

$$
b_{0}>\frac{c}{H_{0}} \frac{n(n-1)}{\sqrt{3 n(n+2)}} .
$$

Since $1 / H_{0}$ is of the order of the age of the Universe, $c / H_{0}$ is of the order of the size of the Universe. This contradicts the physical assumption that $b_{0}$ be small.

The argument we have given is independent of the detailed nature of the internal manifold. For a nonmaximally symmetric space with nonzero curvature, the term $n(n-1) k_{n} / 6 b^{2}$ is replaced by a curvature tensor. By dimensional analysis the curvature tensor varies as $b^{-2}$, where $b$ characterizes its scale. The line of reasoning given above still holds and so, disregarding special choices of $\hat{\rho}$, the internal space must have zero curvature if it is to be physically consistent. For the rest of this sec- 
tion we set $k_{n}=0$.

Another notable feature of the $D$-dimensional theory for $D>4$ is that the evolution of the Universe is governed by equations that are qualitatively different from the usual four-dimensional case. For $n=0$, the Einstein equations reduce to the first two equations of (5.2) and describe Friedmann cosmologies. The evolution of the spacetime scale parameter $a$ is then governed entirely by a first-order differential equation driven by the mass density $\hat{\rho}$ and by the cosmological constant $\Lambda$ if present. In contrast, for the higher-dimensional theories the time development of $a$ is linked to $b$ via a pair of second-order equations and is dictated by $\rho$, by $\hat{\tau}$, by $\Lambda$, and by the manner in which $b$ evolves. For the special case of $n=1$, i.e., $D=5, a$ is governed by a second-order equation with the value of $\rho$ having no effect whatsoever. This special case is considered in more detail in Secs. V C, VD, and $\mathrm{V}$ E below.

\section{B. The variation of $G_{N}$ with time}

For arbitrary $n$, exact solutions to (5.2) are difficult to find. Despite this, it is possible to find phenomenological constraints excluding certain models. We discuss here constraints arising from the time variation of the Newton constant $G_{N}$.

From Eq. (4.15) $G_{N}$ is proportional to $b^{-n}$. Consequently, the dynamical evolution of $b$ means that the Newton gravitational constant varies with time. ${ }^{36,22,23,26,31,37-47}$ Via the first equation in (5.2) we find that in the current epoch ${ }^{10}$

$$
\left.\frac{1}{H} \frac{\dot{G}_{N}}{G_{N}}\right|_{0}=\left\{\begin{array}{l}
\frac{3 n}{n-1} \pm\left[\frac{3 n(n+2)}{(n-1)^{2}}+\frac{3 n(n+2) \Omega_{0}}{(n-1)(n+1)}-\frac{6 n}{n-1} \frac{k}{a_{0}^{2} H_{0}^{2}}\right]^{1 / 2} \text { when } n>1 \\
1-\frac{3 \Omega_{0}}{4}+\frac{k}{a_{0}^{2} H_{0}^{2}} \text { when } n=1
\end{array}\right.
$$

where

$$
\Omega_{0}=8 \pi G_{N} \rho_{0} / 3 H_{0}^{2} .
$$

In the standard cosmological scenario, the ratio $\Omega$ of the mass-energy density to its critical value determines whether the Universe is closed $(\Omega>1)$ or open $(\Omega<1)$. For the higher-dimensional models in Eq. (5.2), $\Omega$ does not serve this role; it is simply a useful dimensionless quantity.

In many of the models, the variation of $G_{N}$ with time exceeds the current experimental upper limit, ${ }^{48}$ given by $\left|\dot{G}_{N} / G_{N}\right|<6 \times 10^{-12} \mathrm{yr}^{-1}$. This bound implies that the right-hand side of Eq. (5.7) must be less than 0.12 if a conservatively low value of $H_{0}=50 \mathrm{~km} \mathrm{~s}^{-1} \mathrm{Mpc}^{-1}$ is used. However, the right-hand side of Eq. (5.7) is of the order of one, unless $\Omega_{0}$ and $a_{0}$ are finely adjusted. For example, if $k=0$ or $c /\left(a_{0} H_{0}\right)<<1, \Omega_{0}$ must fall within approximately $12 \%$ of a critical value $\Omega_{\text {crit }}(n)$ :

$$
\Omega_{0} \approx \Omega_{\text {crit }}(n)(1.0 \pm 0.12),
$$

where

$$
\Omega_{\text {crit }}(n)=\frac{2(n+1)}{n+2} .
$$

The experimental range $e^{49-54}$ for $\Omega_{0}$ is $0.01-1.2$, with some uncertainty on the upper limit due to the possibility of unobserved dark matter. If we take an experimental upper bound of 1.2 then models with $k=0$ are excluded. The same is true for models with $k=1$ because $\Omega_{\text {crit }}$ for $k=1$ is larger than $\Omega_{\text {crit }}$ for $k=0$. The only cosmologies admitted by this experimental upper bound are the ones with $k=-1$, for which $a_{0}$ must be delicately adjusted so that acceptable experimental values of $\Omega_{0}$ yield a sufficiently small value of $\left|\dot{G}_{N} / G_{N}\right|$. Thus, all admissible models have a fine-tuning problem.

\section{The macroscopic scale parameter in five dimensions}

More insight may be gained by examining in detail the simplest model, which has $n=1$. The third equation in (5.2) reduces to ${ }^{10}$

$$
\frac{\ddot{a}}{a}+\left(\frac{\dot{a}}{a}\right)^{2}=-\frac{k}{a^{2}} .
$$

It has the solution

$$
a(t)=\sqrt{-k\left(t-t_{0}\right)^{2}+2 a_{0} \dot{a}_{0}\left(t-t_{0}\right)+a_{0}^{2}} .
$$

Four classes of models may be distinguished. Class (a) has $k=0$; class (b) has $k=1$; class (c) has $k=-1$ and $\dot{a}_{0} / c \leq 1$; and class (d) has $k=-1$ and $\dot{a}_{0} / c \geq 1$. Classes (c) and (d) coincide when $\dot{a}_{0}=c$.

The age $t_{U}$ of the Universe in each model is

class (a): $t_{U}=\frac{1}{2 H_{0}}$,

class (b): $t_{U}=\frac{1}{H_{0}}\left(\frac{\dot{a}_{0}}{c}\right]^{2}\left\{\left[1+\left(\frac{c}{\dot{a}_{0}}\right]^{2}\right]^{1 / 2}-1\right\}$,

class (c): $t_{U}=\frac{1}{H_{0}}\left(\frac{\dot{a}_{0}}{c}\right)^{2}$,

class (d): $t_{U}=\frac{1}{H_{0}}\left(\frac{\dot{a}_{0}}{c}\right]^{2}\left\{1-\left[1-\left(\frac{c}{\dot{a}_{0}}\right]^{2}\right]^{1 / 2}\right\}$.

Here, the deceleration parameter $q_{0}$ is given by

$$
q_{0} \equiv-\frac{\ddot{a}_{0} a_{0}}{\dot{a}_{0}^{2}}=1+\frac{k c^{2}}{\dot{a}_{0}^{2}},
$$

and $H_{0}=45-100 \mathrm{~km} \mathrm{~s}^{-1} \mathrm{Mpc}^{-1}$ is the measured 
value $^{55-65}$ of the Hubble constant. In the standard Friedmann cosmologies $q_{0}=\Omega_{0} / 2$, whereas in the $D=5$ model this relation is replaced by (5.14).

Th age of the Universe for models in classes (a), (b), and $(\mathrm{d})$ is determined from Eq. (5.12) by finding the value of $t$ that renders $a(t)$ zero. For models in class (c), $a(t)$ never vanishes. Instead, $t_{U}$ is taken as the value of $t$ which yields the minimum value for $a(t)$.

Shifting $t$ by $t_{U}-t_{0}$ in Eq. (5.12) allows the time evolution to be expressed in terms of a new variable measuring time from the beginning of the Universe. The solution (5.12) becomes

$$
\frac{a(t)}{a_{0}}=\left\{\begin{array}{l}
\left\{2\left[1+k\left[\frac{c}{\dot{a}_{0}}\right]^{2}\right]^{1 / 2} H_{0} t-k\left(\frac{c H_{0} t}{\dot{a}_{0}}\right]^{2}\right\}^{1 / 2} \text { for classes(a), (b), and (d) }, \\
{\left[1-\left[\frac{\dot{a}_{0}}{c}\right]^{2}+\left(\frac{c H_{0} t}{\dot{a}_{0}}\right]^{2}\right]^{1 / 2} \text { for class (c) } .}
\end{array}\right.
$$

model (a)

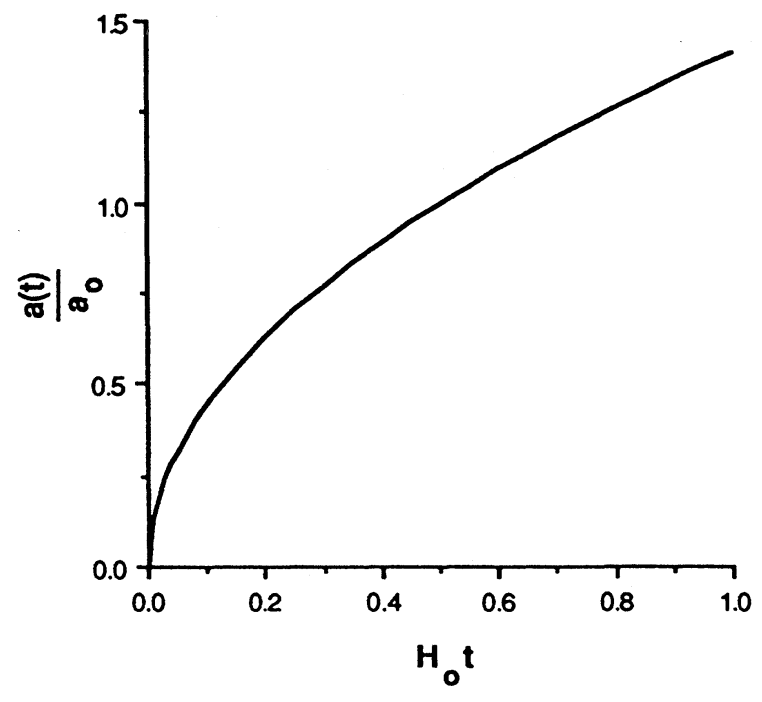

model (c)

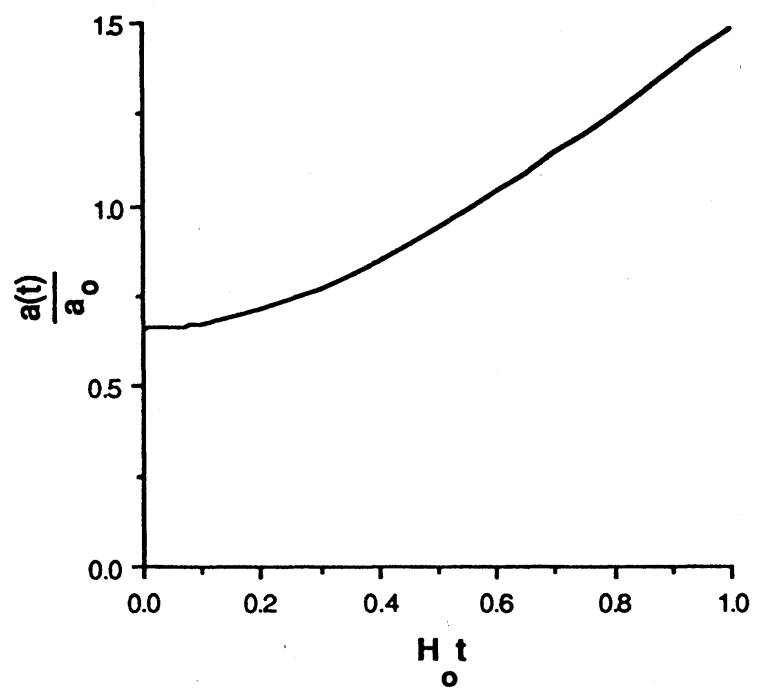

model (b)
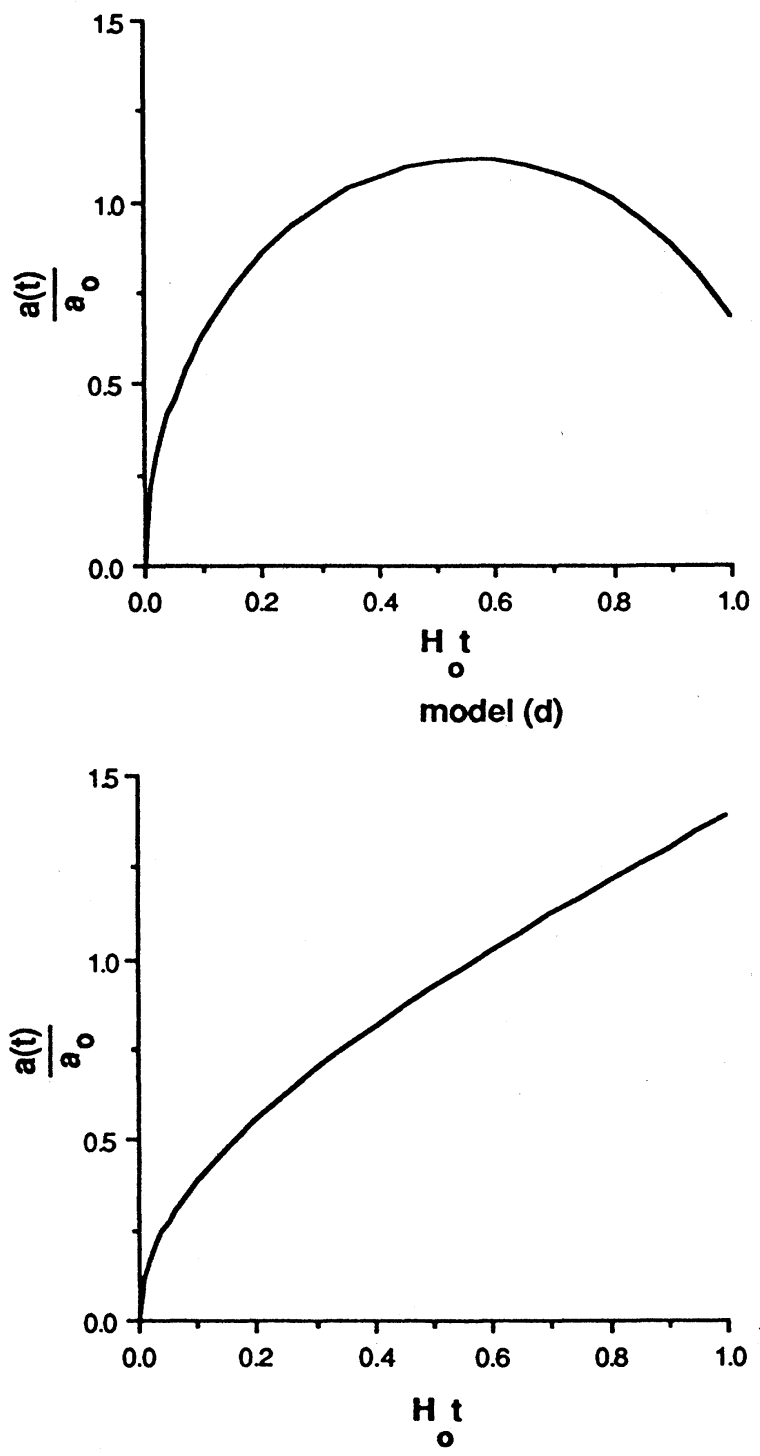

FIG. 1. Evolution of the scale parameter as a function of the Hubble time. (a) The $k=0$ model. (b) The $k=+1$ model with $\dot{a}_{0} / c=0.5$. (c) The $k=-1$ model with $\dot{a}_{0} / c=0.75$. (d) The $k=-1$ model with $\dot{a}_{0} / c=1.5$. 
As can be seen from these equations, the evolution ratio $a(t) / a_{0}$ is a function of $k, H_{0} t$, and $\dot{a}_{0} / c$. In Fig. 1 we display typical plots of $a(t) / a_{0}$ versus $H_{0} t$. Figure 1(a) shows the case of class (a). Its evolution is independent of $\dot{a}_{0} / c$. Figures $1(\mathrm{~b}), 1(\mathrm{c})$, and $1(\mathrm{~d})$ represent plots for models in classes (b), (c), and (d), with values of $\dot{a}_{0} / c$ of 0.5 , 0.75 , and 1.5 , respectively. None of these evolutions is compatible with astronomical data because all yield too small a value for the age of the Universe. The purpose of these plots is to present the solutions qualitatively and pictorially.

\section{Astrophysical phenomenology in five dimensions}

Let us next discuss in more detail the astrophysical phenomenology constraining these five-dimensional models.

Models of type (c) are probably ruled out because measurements of $q_{0}$ favor a positive value, ${ }^{66}$ representing a decelerating universe. That $q_{0}$ is negative for class (c) manifests itself in Fig. 1(c): the graph is concave upward. Furthermore, since $a(0) \neq 0$ the ratio of the size of the Universe at present to its size at incipience is, in general, a number greater than one but of order of unity. To avoid these problems the $q_{0} \rightarrow 0$ limit of the model must be taken. This makes the models in class (c) approach the ones in class (d) in the $q_{0} \rightarrow 0$ limit. The latter are considered in detail below.

The physical age of the Universe $t_{U}$ is known from radioactive dating, ${ }^{67}$ the age of globular clusters, ${ }^{55,64,68,69}$ the age of the chemical elements, ${ }^{70}$ and the age of Earth ${ }^{71}$ to be $1.5-1.9 \times 10^{10} \mathrm{yr}$. This value is sufficiently well established to exclude the models in Fig. 1, at least for the values of $\dot{a}_{0} / c$ chosen. For Figs. $1(\mathrm{a}), 1(\mathrm{~b}), 1(\mathrm{c})$, and $1(\mathrm{~d})$, $t_{U}$ is $0.5 / H_{0} \approx 0.89 \times 10^{10} \mathrm{yr}, 0.31 / H_{0} \approx 0.55 \times 10^{10} \mathrm{yr}$, $0.56 / H_{0} \approx 1.01 \times 10^{10} \mathrm{yr}$, and $0.57 / H_{0} \approx 1.02 \times 10^{10} \mathrm{yr}$, respectively, where the value $H_{0}=h_{0} \equiv 55 \mathrm{~km} \mathrm{~s}^{-1} \mathrm{Mpc}^{-1}$ has been used.

The constraints coming from $t_{U}$ can be more systematically analyzed by noting that Eq. (5.13) implies

$$
\begin{aligned}
& t_{U}=\frac{1}{2 H_{0}} \text { for class (a), } \\
& 0 \leq t_{U} \leq \frac{1}{2 H_{0}} \text { for class }(\mathrm{b}) \\
& 0 \leq t_{U} \leq \frac{1}{H_{0}} \text { for class }(\mathrm{c}) \\
& \frac{1}{2 H_{0}} \leq t_{U} \leq \frac{1}{H_{0}} \text { for class }(\mathrm{d})
\end{aligned}
$$

Since $\left(2 H_{0}\right)^{-1}=0.89 \times 10^{10}$ yr when $H_{0}=h_{0}$, classes (a) and (b) are incompatible with experiment. A higher value of $H_{0}$ yields even smaller values of $t_{U}$ for these models, thereby augmenting the disagreement with experiment.

Explicit solutions for the $n \geq 2$ models are also likely to provide constraints from $t_{U}$.

The only consistent class of five-dimensional models appears to be (d). Furthermore, for this case $\dot{a}_{0} / c$ must be fine-tuned to be slightly greater than 1 . For the quoted value of $H_{0}$, one needs $1 \leq \dot{a}_{0} / c \leq 1.019$ to have $t_{U}>1.5 \times 10^{10} \mathrm{yr}$. The corresponding values of $q_{0}$ range from 0 to 0.037 . For $q_{0}=0$, the solution of the $k=-1$ model simplifies to $a(t) / a_{0}=H_{0} t$; i.e., the scale parameter grows linearly with time.

We find that standard Friedmann cosmologies can be numerically reproduced in the class (d) of models by suitably adjusting parameters. Typically, the only significant difference occurs during early times before the first $4 \times 10^{9} \mathrm{yr}$.

Let us compare a realistic model of type (d) to a standard $k=-1$ Friedmann cosmology. Fixing $H_{0} t_{U}$ specifies the dependence of $a(t) / a_{0}$ on $H_{0} t$ in both cases. For the five-dimensional model, this follows from Eq. (5.13): $t_{U} / H_{0}$ fixes $\dot{a}_{0} / c$ thereby determining Eq. (5.15). For the standard model, $q_{0}$ is determined by $t_{U} H_{0}$ via the equation $^{72}$

$t_{U} H_{0}=\left(1-2 q_{0}\right)^{-1}-q_{0}\left(1-2 q_{0}\right)^{-3 / 2} \operatorname{arccosh}\left(\frac{1}{q_{0}}-1\right)$,

and then the dependence of $a(t) / a_{0}$ on $H_{0} t$ is given implicitly by

$$
\begin{aligned}
& \frac{a(t)}{a_{0}}=\frac{q_{0}}{1-2 q_{0}}(\cosh \psi-1), \\
& H_{0} t=\frac{q_{0}}{\left(1-2 q_{0}\right)^{3 / 2}}(\sinh \psi-\psi) .
\end{aligned}
$$

Setting the age of the Universe to be $0.95 \mathrm{H}_{0}^{-1}$ $\approx 1.7 \times 10^{10}$ yr with $H_{0}=h_{0}$, we find virtually no difference between the two models. At $t=0.1 t_{U}, a(t) / a_{0}$ is about $10 \%$ larger for the five-dimensional case, at $t=0.2 t_{U}$ it is $5 \%$ larger, at $t=0.4 t_{U}$ there is a $1 \%$ difference, and beyond $t=0.6 t_{U}$ the two differ by less than $0.2 \%$. See Fig. 2 .

\section{E. The internal scale parameter in five dimensions}

So far we have largely been concerned with the evolution and experimental properties of the macroscopic scale parameter $a$. Next, we consider the internal scale parameter $b$ in the five-dimensional model.

With an energy density comprised of both matter and radiation, $\rho=\rho_{m}+\rho_{r}$, where $\rho_{m}$ and $\rho_{r}$ independently satisfy Eq. (5.3), it is possible to find an exact solution for $b(t)$. The first of Eqs. (5.2) becomes

$$
\left(\frac{\dot{a}}{a}\right)^{2}+\frac{\dot{a}}{a} \frac{\dot{b}}{b}=-\frac{k}{a^{2}}+\frac{8 \pi G_{D}}{3 b}\left(\frac{\rho_{m 0} a_{0}^{3}}{a^{3}}+\frac{\rho_{r 0} a_{0}^{4}}{a^{4}}\right) .
$$

Its solution is

$$
\frac{b}{b_{0}}=\left(1-\frac{3 \Omega_{r 0}}{4 q_{0}}\right) \frac{\dot{a}}{\dot{a}_{0}}+\frac{3 \Omega_{m 0} \dot{a}_{0}}{4 a}\left(t-t_{0}\right)+\frac{3 \Omega_{r 0}}{4 q_{0}},
$$




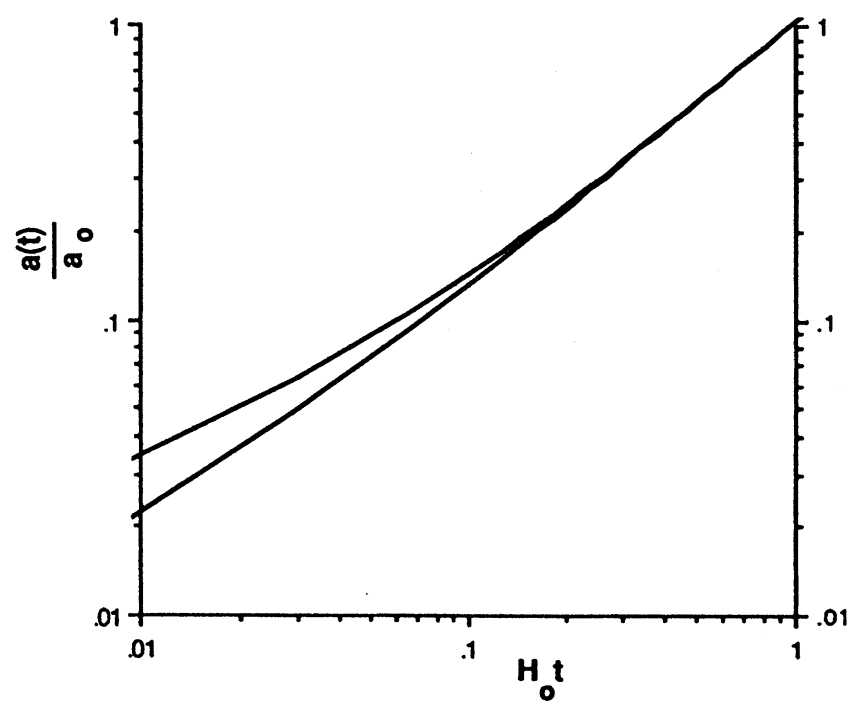

FIG. 2. Comparison of a standard cosmology with a fivedimensional type-(d) model. The lower curve is that of the standard cosmology. Both curves have $t_{U}=0.95 / H_{0}$.

where $a$ and $\dot{a}$ are to be taken from Eq. (5.12), where $q_{0}$ is given in Eq. (5.14), and where

$$
\Omega_{m 0}=\frac{\rho_{m 0}}{\rho_{c}}, \quad \Omega_{r 0}=\frac{\rho_{r 0}}{\rho_{c}}, \rho_{c}=\frac{3 H_{0}^{2}}{8 \pi G_{N}} .
$$

An interesting feature of Eq. $(5.20)$ is that $b(t)$ contracts as $a(t)$ expands with $t$. This mechanism might explain why the scale of the internal dimensions is small. ${ }^{73}$ It simplifies the understanding of the behavior of $b(t)$ to set $\Omega_{r 0}=0$, which is consistent with astrophysical measurements shown that $\Omega_{r 0} \ll 1$. One finds

$$
\frac{b}{b_{0}}=\frac{a_{0}}{a}\left\{1+\left[\frac{3}{4} \Omega_{m 0}-k\left[\frac{c}{\dot{a}_{0}}\right]^{2}\right] H_{0}\left(t-t_{U}\right)\right\} \text {. }
$$

Since for small $t$,

$$
\frac{a}{a_{0}} \sim c_{2} \sqrt{H_{0} t}
$$

where

$$
c_{2}^{2}=2\left[1+k\left(\frac{c}{\dot{a}_{0}}\right]^{2}\right]^{1 / 2},
$$

it follows that

$$
\frac{b}{b_{0}} \sim \frac{c_{1}}{c_{2}} \frac{1}{\sqrt{H_{0} t}}
$$

where

$$
c_{1}=1-H_{0} t_{U}\left[\frac{3}{4} \Omega_{m 0}-k\left(\frac{c}{\dot{a}_{0}}\right)^{2}\right] .
$$

Take $c_{1}>0$; if this is not the case, $t_{U}$ must be determined by where $b$ vanishes rather than $a$. Equations (5.23) and (5.25) show that, while $a(t)$ grows with $t, b(t)$ decreases with $t$. If we require that the $a$ and $b$ scales be comparable at some early time $t_{e}$, i.e., $b\left(t_{e}\right)=a\left(t_{e}\right)$, then $b_{0} / a_{0}=\left(c_{2}^{2} / c_{1}\right) H_{0} t_{e}$. Since $t_{U} \sim 1 / H_{0}$, this shows that $b_{0} / a_{0} \sim t_{e} / t_{U}$. The ratio $t_{U} / t_{e}$ is likely to be many orders of magnitude if $a\left(t_{e}\right)$ and $b\left(t_{e}\right)$ are microscopic at time $t_{e}$. Consequently, the scale $b_{0}$ of the internal dimensions today is significantly smaller than $b\left(t_{e}\right)$.

Note that the equations and the physics they contain cannot be trusted at the earliest stages of the Universe. However, one knows that the equations hold after a certain early time beyond which $b(t)$ grows smaller with time in the manner prescribed by Eq. (5.25).

Although the discussion in the previous paragraph holds for the $n=1$ model, it should be a feature for realistic models when $n \geq 2$. Since $\dot{b} / b$ and $\dot{a} / a$ enter in Eq. (5.2) in a similar manner, dimensional analysis implies that typically $|\dot{b} / b| \sim|\dot{a} / a|$. From this one concludes $b_{0} / b\left(t_{e}\right) \sim\left[a_{0} / a\left(t_{e}\right)\right]^{p}$ for some power $p$, where $t_{e}$ is some early time at which $a$ and $b$ were of comparable size. If $p>0$ then $b_{0} \sim a_{0}^{p}$ and $b_{0}$ is of macroscopic size, which is not experimentally viable. If $p<0$ then $b(t)$ shrinks as $a(t)$ grows. The factor by which $a$ to the $p$ th power has grown since early times should be the factor by which $b$ has decreased. This is what we found above for the $n=1$ case for which $p=1$ and the constants $c_{1}$ and $c_{2}$ are of order unity.

\section{F. Inflation for arbitrary dimensions}

In an inflationary scenario ${ }^{74-76}$ for a higherdimensional theory with a linear $R$ term, the behavior of $b$ with time is problematic. The physics of inflation is mimicked by a time-dependent cosmological constant which is positive during the early stages of the Universe and zero later. The positive cosmological constant causes the Universe to expand rapidly. Later, when it becomes zero, the evolution proceeds in the conventional manner.

The basic problem with higher-dimensional theories is that, typically, if $a$ inflates so does $b$. The internal dimensions would then be of cosmological size, even taking into account the shrinking mechanism discussed in Sec. VE (Ref. 77). The effect is seen as follows. When the cosmological constant $\Lambda$ is positive, it dominates the right-hand sides of Eqs. (5.2). One can verify that

$$
\begin{aligned}
& a(t)=a(0) \exp \left[\left(\frac{2 \Lambda}{(n+2)(n+3)}\right)^{1 / 2} t\right], \\
& b(t)=b(0) \exp \left[\left(\frac{2 \Lambda}{(n+2)(n+3)}\right)^{1 / 2} t\right]
\end{aligned}
$$

is then a solution. The scales $a(t)$ and $b(t)$ grow together exponentially at the same rate. Although other solutions can be found, e.g., changing the sign of both exponents in Eq. (5.27), none of them involve exponential growth in $a(t)$ and thus none correspond to an inflationary scenario. In short, inflation in a higher-dimensional theory with only a linear $R$ term renders $b_{0}$ of cosmologi- 
cal size. Higher powers of $R$ in the action may avert this difficulty.

\section{DISCUSSION AND CONCLUSIONS}

In this paper we investigated consequences of tensorinduced spontaneous Lorentz-symmetry breaking in higher-dimensional theories. We also studied possible phenomenological gravitational effects both with and without the presence of tensor-induced breaking and identified particular gravitional phenomena placing constraints on such theories. The ideas were developed through a string-inspired model. The results obtained are relevant not only to strings but also to Kaluza-Klein and $D>4$ supergravity theories.

The strongest constraint comes from the observation that a localized mass distribution must generate a fourdimensional metric of the Eddington-Robertson form, with parameters that are well-established experimentally. Higher-dimensional theories can yield parameters in conflict with the observed data. Our analysis is based on perturbative calculations in higher-dimensional theories with purely an Einstein $R$ term, with or without tensorinduced spontaneous Lorentz-symmetry breaking. However, the conclusions should be independent of terms in the Lagrangian of higher powers in $R$, since by dimensional analysis these do not affect the leading largedistance behavior.

For theories with spontaneous Lorentz-symmetry breaking induced by the vacuum expectation value of a tensor field, features of the compactified dimensions were deduced using methods of algebraic topology. We found that for our string-inspired model the compactified manifold must have zero Euler characteristic and nonzero first Betti number. Indeed, the compactified manifold must be a fiber bundle over a circle.

Further phenomenological constraints arise from cosmological considerations. We explored model universes for pure Einstein theories in higher dimensions. We found that the compactified dimensions are naturally flat if they are small. Many theories can be excluded on the basis of disagreement with experimental knowledge of quantities such as the age of the Universe and the variation of the Newton gravitational constant with time. Higher-dimensional models mimicking the standard cosmology and satisfying observational constraints can only be established if various parameters are fine-tuned. More precise measurements of the Hubble expansion constant, the deceleration parameter, and the mass density of the Universe could therefore eliminate these possibilities also.

Disregarding technical details and potential complications, the key reason for the phenomenological difficulties may be viewed as follows. The modifications to Einstein gravity are a consequence of the long-range propagation of massless modes of the Lorentz-scalar components $h_{j k}$, $j, k \geq 4$, of the higher-dimensional components of the metric $h_{\mu \nu}$. Like the graviton, these modes couple weakly and cannot be directly detected. Nonetheless, they cause subtle effects: without modifying Newton gravity they induce measurable deviations from Einstein gravity and changes in cosmological parameters.

We are thus faced with the following result: the perturbative sector of many higher-dimensional theories is excluded on the basis of current experimental observations. Since some of the most attractive theoretical proposals for unification and quantum gravity are based on the presence of extra dimensions and can have massless models of the type discussed, circumventing this result is desirable. In the remainder of this section we mention some possibilities that may be relevant to this issue.

We remark that massless Lorentz-scalar modes might be avoided altogether in certain compactification schemes. However, an arbitrary manifold is not admissible: it must obey the equations of motion and be stable under small fluctuations. Compactifications often have zero modes; for example, it is common in Kaluza-Klein, supergravity, and superstring theories to consider Ricciflat manifolds, which typically have zero modes. Zero modes occur in torodial compactifications, including the original five-dimensional Kaluza-Klein model, ${ }^{1,2}$ and generically occur in models based on Calabi-Yau manifolds. ${ }^{15}$

Granting the existence of such modes in a model, the phenomenological difficulties may nonetheless be obviated if a mechanism can be found preventing or damping their long-range propagation. The simplest solution is to give them masses. However, doing so explicitly is not permitted because it violates general coordinate invariance. This is similar to gauge theories for particle physics where the vector gauge bosons cannot be given masses by hand due to gauge invariance. In such theories, masses can be generated for vector gauge bosons via the Higgs mechanism. This fact was our primary motivation for the investigation of the gravitational version of the Higgs effect. However, as demonstrated in Sec. III, this effect neither provides masses for components of the graviton nor destabilizes flat higher-dimensional spacetime.

Let us consider further the analogy to non-Abelian gauge theories. What mechanisms exist for mass generation other than the Higgs effect? The pure SU(3) nonAbelian gauge theory provides an example. Perturbatively, it contains eight massless gluons, yet the physical spectrum is believed to consist of massive glueballs states. A single gluon cannot be microscopically isolated because of confinement. Effectively, the mass of a gluon increases as it moves away from a bound state. Thus, the propagation of the massless modes of the perturbative sector is inhibited at macroscopic distances.

Perhaps an analogous mechanism occurs in string theory. String interactions are highly nonlinear and involve an infinite number of particle modes. Furthermore, there are arguments based on the effective dilaton potential $^{78}$ indicating a need for strong coupling in string theories. If string interactions become strong at large distances, massive bound states analogous to glueballs might form. If these states include the higherdimensional components of the metric then the latter essentially decouple at large distances and the phenomenological difficulties are avoided. Current knowledge suggests a gravitational version of confinement is unlikely 
to occur in Kaluza-Klein or supergravity theories. Only the primitive understanding of nonlinear effects among the infinite number of degrees of freedom prevents us from excluding this idea in string theory.

Another possibility is that bound states occur directly as a result of short-distance effects. The gravitational potential in $D$ flat dimensions varies as $r^{3-D}$ and hence at short distances becomes stronger as $D$ increases. If the components of the metric associated with the extra dimensions form a small but massive bound state, their propagation at large distances will be inhibited.

Even presupposing a mechanism for the damping of higher-dimensional components of the metric, a second issue of importance arises. String theories when treated perturbatively yield interactions of the type seen in nature: namely, four-dimensional gravitational and nonAbelian gauge interactions. It is therefore desirable to keep certain pieces of the perturbative sector intact. The asymmetry problem then occurs: one must understand why only one part of the theory involves a damping mechanism.

Grand unified theories provide an analogue system in particle physics. They incorporate the strong and electroweak interactions. The former is strongly coupled while the latter is weak. The former occurs in bound states while the latter has massless propagating photons. Hence, grand unified theories display the desired asymmetry. In general, the grand-unification gauge group $G$ is broken to a non-Abelian subgroup $H$ and one or more $\mathrm{U}(1)$ factors. The $\mathrm{U}(1)$ gauge bosons remain massless and generate long-range forces. The non-Abelian gauge bosons are confined and form bound states. Following this analogy, the four-dimensional degrees of freedom in string theory should be like the $U(1)$ gauge bosons while the higher-dimensional degrees of freedom should be like the $H$ gauge bosons. The explicit implementation of this possibility is an interesting theoretical problem.

The key to the asymmetric requirement must be a symmetry-breaking mechanism. While tensor-induced spontaneous Lorentz-symmetry breaking does not directly solve the mass generation problem, it may nonetheless provide the needed impetus for the asymmetry. In this regard, string theory is favored over other higherdimensional theories.

Higher-dimensional strings may be naturally unstable and may spontaneously break to lower-dimensional spacetimes in one or more stages and by one or more mechanisms other than tensor-induced breaking. The reason why $D=4$ is selected as the critical dimension is unclear. Special features of $D=4$ might play a role: $D=4$ is an upper critical dimension for many systems of statistical mechanics; and for $D>4$ renormalizability in certain field theories and asymptotic freedom in nonAbelian gauge theories are both lost.

It is also possible that some exotic and instrinsically stringy compactification scheme avoids the phenomenological problems we have discussed. The generation of masses for the higher-dimensional components of the metric in a superstring theory might be linked to the process that breaks supersymmetry and generates fermion masses.
In conclusion, there can be observable gravitational effects due to the extra dimensions even when these form a manifold of microscopic size. The experimental absence of these effect poses a challenge to many higherdimensional theories.

We have seen that compactification of the extra dimensions can by itself be insufficient to ensure a phenomenologically viable theory. Some damping mechanism must be found for any massless modes of the components $h_{j k}$, $j, k \geq 4$, of the graviton to prevent their long-range propagation and corresponding effects. This damping problem is crucial for agreement with precise experimental tests of nonleading effects in Einstein gravity: A mechanism for mass generation might be related to a bound-state problem or to a kind of dimension confinement.

Phenomenological viability of string theories thus suggests that certain degrees of freedom are in a nonperturbative regime while others, such as the physical fourdimensional components of the graviton, are manifested in the perturbative sector. This poses a second problem: find a way to generate the needed asymmetry. In this regard, tensor-induced spontaneous Lorentz-symmetry breaking may play a role.

\section{ACKNOWLEDGMENTS}

We enjoyed particularly beneficial conservations with J. Davis. We thank F. Cooper, D. Z. Freedman, M. Roček, and B. Sakita for stimulating questions. We also thank R. Bluhm, A. Edmonds, A. Hendry, D. Lichtenberg, and $\mathrm{C}$. Livingston for discussions and $\mathrm{K}$. Bardakçi, M. Burkhead, S. Deser, R. Durisen and, especially, S. Mufson for suggesting useful references. Some computer equipment used is owned by the Physics Department of The City College of New York. This work was supported in part by the United States Department of Energy under Contracts Nos. DE-AC02-84ER40125 and DE-AC0283ER40107.

\section{APPENDIX A}

This appendix proves that a manifold $M^{n}$ satisfying conditions (i) and (ii) of Sec. III C is a fiber bundle over $S^{1}$. The proof is due to Davis.

The first step of the proof is to use the conditions to define a map $f: M^{n} \rightarrow S^{1}$ such that $f_{*}(a)(m) \neq 0$ for all $m \in M^{n}$. The map is relevant because it can be used to construct a bundle $B$ over $S^{1}$. Here, we treat $\hat{a}$ as the one-form obtained from a using the metric.

Condition (ii) provides a closed one-form $\widehat{a} \in H^{1}\left(M^{n}, \mathbb{R}\right)$. The first cohomology group $H^{1}\left(M^{n}, \mathbb{R}\right)$ over the reals is related to the first cohomology group $H^{1}\left(M^{n}, \mathbb{Z}\right)$ over the integers via the universal-coefficient theorem: ${ }^{79} H^{1}\left(M^{n}, \mathbb{R}\right) \cong H^{1}\left(M^{n}, \mathbb{Z}\right) \otimes \mathbb{R}$. We can replace $\widehat{a}$ with a closed one-form $\omega \in H^{1}\left(M^{n}, \mathbb{Z}\right)$, given explicitly as follows. Let the set $\left\{\omega^{j}\right\}$ be a basis for $H^{1}\left(M^{n}, \mathbb{Z}\right)$, where $j=1, \ldots, b_{1}$, with $b_{1}$ the first Betti number of $M^{n}$. Given a basis $\left\{c_{j}\right\}$ of the one-cycles for $H_{1}\left(M^{n}, \mathbb{Z}\right)$ modulo torsion, the $\omega^{j}$ can be chosen to satisfy $\int_{c_{k}} \omega^{j}=\delta_{k}^{j}$ by the first de Rham existence theorem. ${ }^{80}$ Then, $\hat{a} \in H^{1}\left(M^{n}, \mathbb{R}\right)$ may be written as $\hat{a}=a_{j} \omega^{j}+d \lambda$, 
where $a_{j} \in \mathbb{R}$ and repeated indices are summed. Define $\omega^{\prime}=r_{j} \omega^{J}+d \lambda$, where the $r_{j}$ are rational numbers chosen sufficiently close to the $a_{j}$ so that $\omega^{\prime}(\mathbf{a})>1-\epsilon \equiv \kappa>0$. This is possible because $\hat{a}(\mathbf{a})=1$. Then, define the desired closed form $\omega$ by multiplying $\omega^{\prime}$ with an integer $N: \omega=n_{j} \omega^{j}+N d \lambda \in H^{1}\left(M^{n}, \mathbb{Z}\right), \quad$ where $n_{i} \in \mathbb{Z} \quad$ and $\omega(\mathbf{a})>N \kappa>0$.

The map $f: M^{n} \rightarrow S^{1}$ can now be defined. Fix a point $p \in M^{n}$. Let $q$ be another point in $M^{n}$ and let $\gamma_{p q}$ be any curve from $p$ to $q$. Define $f(q)=\int_{\gamma_{p q}} \omega \bmod \mathbb{Z}$. The mod $\mathbb{Z}$ restriction ensures $0 \leq f(q)<1$. Note that $f_{*}(\mathbf{a})$ is uniformly bounded away from zero because $\omega(\mathbf{a})>N \kappa>0$.

Let us check that $f$ does not depend on the specific path $\gamma_{p q}$ used in the construction. Let $\gamma_{p q}^{\prime}$ be another path from $p$ to $q$. Then the difference $\gamma_{p q}^{\prime}-\gamma_{p q}$ is a closed path in $M^{n}$. Such a path can be written as $\gamma_{p q}^{\prime}-\gamma_{p q}=m^{j} c_{j}+\partial E+\tau$, where $\partial E$ is a boundary and $\tau$ is a torion term, i.e., $m \tau=\partial F$ for some $m \in \mathbb{Z}$ and for some boundary $\partial F$. An integral of $\omega$ over $\tau$ vanishes because $\int_{\tau} \omega=(1 / m) \int_{m \tau} \omega=(1 / m) \int_{\partial F} \omega$, which is zero because $\omega$ is closed. Therefore, the difference $f(q)-f\left(q^{\prime}\right)=m^{j} n_{j}$ is an integer. However, an integer ambiguity leaves $f$ unchanged because it is defined mod $\mathbb{Z}$.

The function $f$ may be used to construct the fiber bundle over $S^{1}$ defined in Eq. (3.5). The bundle space $B$ is the manifold $M^{n}$ and the base is $S^{1}$ viewed as the interval $[0,1]$ with the end points identified. The fiber space $N^{n-1}$ is taken as $f^{-1}(0)$. It is a manifold because $f_{*} \neq 0$ and 0 is a regular point. ${ }^{81}$

To complete the definition of the bundle we must specify the monodromy $h$. Let $\Gamma_{n} \in M^{n}$ be the integral curve for the vector field a starting at some fixed point $n \in f^{-1}(0)$. Consider $f(c)$ for $c \in \Gamma_{n}$ as $c$ moves along the integral curve. Starting at 0 , the value of $f(c)$ moves along the unit interval without backtracking because $f_{*}(\mathbf{a})$ is bounded away from zero. Therefore, $f(c)$ increases until it attains $1 \equiv 0 \bmod \mathbb{Z}$. Let $c^{\prime} \in \Gamma_{n}$ be the first value attained by $c$ for which $f$ is reset to zero. The monodromy is then defined by $h(n)=c^{\prime}$. See Fig. 3 .

The monodromy $h$ is a diffeomorphism of $f^{-1}(0)$ into $f^{-1}(0)$. It is continuous and smooth because the construction involves continuous, smooth functions. It is one to one because two initial points $n$ and $n^{\prime}$ of $f^{-1}(0)$ cannot be mapped to the same point, as integral curves do not cross. It is onto because flowing backward along $\Gamma$ yields $h^{-1}$.

To complete the proof of topological equivalence between the manifold $M^{n}$ and the $S^{1}$ bundle, we must construct a homeomorphism

$$
H: \quad M^{n} \cong \frac{N^{n-1} \times I}{(n, 0) \sim(h(n), 1)} \equiv B,
$$

where $I$ is the interval $[0,1]$. We begin by defining a homeomorphism $H^{\prime}$ from $M^{n}$ onto $N^{n-1} \times[0,1)$. Subsequently, the behavior at the boundary of $I$ is treated.

Let $m$ be a point of $M^{n}$, and set $t=f(m)$. Consider the integral curve $\Gamma$ of a in $M^{n}$ passing through $m$. Flow back to the point $n$ determined by $f(n)=0$. The desired map is then defined by $H^{\prime}(m)=(n, t)$.
The map $H^{\prime}$ is smooth on $M^{-1} \backslash f^{-1}(0)$ because it is defined in terms of smooth functions. It is onto because $(n, 0)$ is in $f^{-1}(0)$ and the integral $\int_{\gamma_{p q}} \omega$ along an integral curve $\gamma_{p q}$ of a increases without bound, so that $(n, t)$ is eventually attained for all $t>0$. It is also one to one, which can be seen as follows. Suppose $m_{1}$ and $m_{2}$ are two points of $M^{n}$ with $H^{\prime}\left(m_{1}\right) \equiv\left(n_{1}, t_{1}\right)$ $=H^{\prime}\left(m_{2}\right) \equiv\left(n_{2}, t_{2}\right)$. From $n_{1}=n_{2}$ and the uniqueness of integral curves, $m_{1}$ and $m_{2}$ are on the same integral curve. By the monotonicity of the integral along the integral curve the point $m$ at which $f(m)=t_{1}=t_{2}$ is unique. Thus, $m_{1}=m=m_{2}$.

Consider next the end points of $I$. Let $H$ be the composition of $H^{\prime}$ and the bijection $N^{n-1} \times[0,1) \rightarrow B$. The points $(n, 0)$ and $(h(n), 1)$ correspond to the same point in $M^{n}$. The map $H$ is continuous because of this equivalence relation. It is the homeomorphism $H$ of Eq. (A1).

We have thus established that $M^{n}$ may be viewed topologically as a fiber bundle over $S^{1}$. This is the theorem quoted as Eq. (3.5).

A further result follows. Given the $S^{1}$ bundle $B$, a
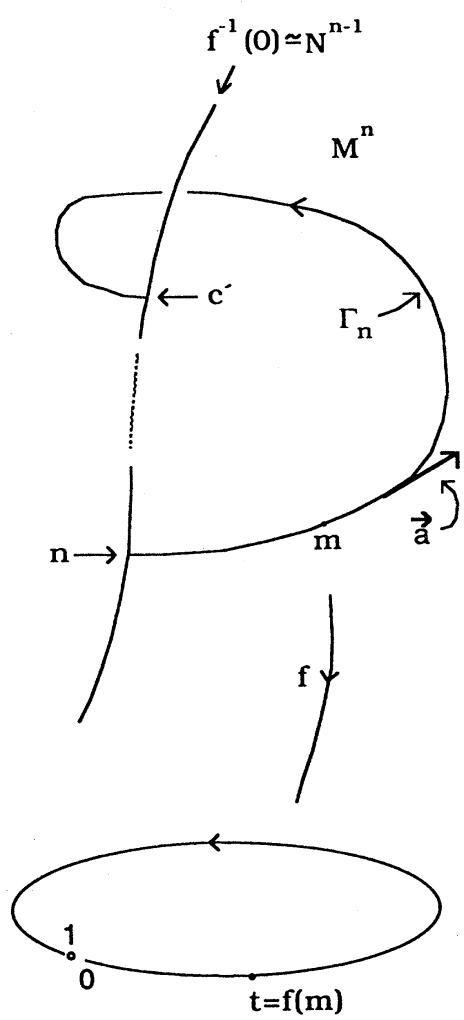

FIG. 3. Representation of the ideas in Appendix A. The space $M^{n}$ is projected onto $[0,1)$ by the map $f$. The fiber $f^{-1}(0)$ is $N^{n-1}$. A point $m \in M^{n}$ determines a value $t$ in $[0,1)$ via $f$ and a point $n \in N^{n-1}$ via the integral curve $\Gamma_{n}$ of a passing through $m$. The integrals may begin and end in different components if $N^{n-1}$ is multiply connected. This possibility is symbolically indicated by the dotted line. 
nowhere-vanishing vector field a and a metric $g$ on $M^{n}$ may be established such that the one-form $\hat{a}$ constructed from a using $g$ is closed. The vector may be taken as $\mathbf{a}=H_{*}^{-1}(0,1)$ in the tangent space at every point. The vector field $(0,1)$ is well defined on the tangent space of $B$ because it is compatible with the monodromy $h$. The metric can be constructed via partitions of unity, as we show next.

Let $f^{(1)}$ be a smooth function on $I$ that is unity in the neighborhood of $1 / 2$ but that vanishes for neighborhoods of 0 and 1. Let $f^{(2)}=1-f^{(1)}$. The system $f^{(1)}$ and $f^{(2)}$ forms a partition of unity for $I$. Let $g^{(1)}$ be a factorized metric $\left(g_{N}, 1\right)$ on $N^{n-1} \times(0,1)$, i.e., $g_{N}$ is any metric on $N^{n-1}$ and 1 is the unit metric on $I$. Define

$$
\begin{aligned}
& F: B \backslash\left(N^{n-1} \times\{1 / 2\}\right) \rightarrow N^{n-1} \times(0,1) \\
& (n, t) \mapsto\left\{\begin{array}{l}
(n, t+1 / 2) \text { for } t<1 / 2 \\
\left(h^{-1}(n), t-1 / 2\right) \text { for } t>1 / 2 .
\end{array}\right.
\end{aligned}
$$

Use $F$ to construct the metric $g^{(2)}$ at a point $p \in B \backslash\left(N^{n-1} \times\{1 / 2\}\right)$ from the metric $g^{(1)}$ at the point $F_{*}(P)$ via the condition

$$
\left.\mathbf{v}_{1} \cdot \mathbf{v}_{2}\right|_{g^{(2)}(p)}=\left.F_{*}\left(\mathbf{v}_{1}\right) \cdot F_{*}\left(\mathbf{v}_{2}\right)\right|_{g^{(1)}\left(F_{*}(p)\right)},
$$

where $\mathbf{v}_{1}$ and $\mathbf{v}_{2}$ are tangent vectors at $p$. Then, $g^{\prime}=f^{(1)} g^{(1)}+f^{(2)} g^{(2)}$ is a metric on $B$. It respects the equivalence relation $(n, 0) \sim(h(n), 1)$ because $F(n, 0)$ $=F(h(n), 1)$.

The metric $g$ on $M^{n}$ can be found from $g^{\prime}$ using the homeomorphism $H$ of Eq. (A1). Given $\mathbf{v}_{1}$ and $\mathbf{v}_{2}$ in the tangent space of $M^{n}$, define

$$
\left.\mathbf{v}_{1} \cdot \mathbf{v}_{2}\right|_{g(m)}=\left.H_{*}\left(\mathbf{v}_{1}\right) \cdot H_{*}\left(\mathbf{v}_{2}\right)\right|_{g^{\prime}(H(m))} .
$$

Then,

$$
\begin{aligned}
\left.\mathbf{a} \cdot \mathbf{a}\right|_{g} & =\left.H_{*}\left(H_{*}^{-1}(0,1)\right) \cdot H_{*}\left(H_{*}^{-1}(0,1)\right)\right|_{g^{\prime}} \\
& =\left.(0,1) \cdot(0,1)\right|_{g^{\prime}} \\
& =1 .
\end{aligned}
$$

As the metric is factorized, the one-form $\hat{a}$ associated with $\mathbf{a}$ is given by the pullback $\hat{a}=H^{*}(d \theta)$ of $d \theta$ in $B$, where $d \theta$ is the standard one-form on the $I$ factor in $B$. It follows from $d d \theta=0$ that $\hat{a}$ is closed, $d \hat{a}=0$. In addition,

$$
\begin{aligned}
\widehat{a}(\mathbf{a}) & =H^{*}(d \theta)\left(H_{*}^{-1}(0,1)\right) \\
& =d \theta H_{*}\left(H_{*}^{-1}(0,1)\right) \\
& =d \theta(0,1)=1
\end{aligned}
$$

\section{APPENDIX B}

This appendix treats the statistical mechanics of an ideal, possibly relativistic gas in $\mathbb{R}^{3} \times M^{n}$. Here, $M^{n}$ is a compactified internal space taken for simplicity to be an $n$-torus $T^{n} \cong S^{1} \times \cdots \times S^{1}$, with the length of the $k$ th circle being $L_{k}$. Replace $\mathbb{R}^{3}$ by a large but finite box of volume $V_{(3)}=L^{3}$. We are interested in the thermodynamic limit in which $V_{(3)} \rightarrow \infty$ and $N \rightarrow \infty$ with $\rho=N / V_{(3)}$ and all $L_{k}$ fixed, where $N$ is the number of particles in the system and $\rho$ is the three-dimensional number density.

The wave functions for the large box are $\sin \left(\pi n_{1} x / L\right) \sin \left(\pi n_{2} y / L\right) \sin \left(\pi n_{3} x / L\right)$, where $n_{i}$ are positive integers. The corresponding momenta are $p_{i}=\pi \hbar n_{i} / L$. The wave functions on the $k$ th circle are one, $\sin \left(2 \pi m_{k} \theta_{k} / L_{k}\right)$, and $\cos \left(2 \pi m_{k} \theta_{k} / L_{k}\right)$, $m_{k}=1,2, \ldots$. The possible values of the internal momenta are zero and $p_{k}=2 \pi \hbar m_{k} / L$, the latter having a degeneracy factor of $2: g(0)=1, g\left(m_{k}\right)=2$ for $m_{k} \geq 1$.

The partition function $Z_{1}$ for a single particle system is

$$
\begin{aligned}
Z_{1}= & {\left[\prod_{i=1}^{3} \sum_{n_{i}=1}^{\infty}\right]\left[\prod_{k=1}^{n} \sum_{m_{k}=0}^{\infty} g\left(m_{k}\right)\right] } \\
& \times \exp \left[-\beta E\left(n_{1}, n_{2}, n_{3}, m_{1}, m_{2}, \ldots, m_{n}\right)\right],
\end{aligned}
$$

where

$$
\begin{aligned}
& E\left(n_{1}, n_{2}, n_{3}, m_{1}, m_{2}, \ldots, m_{n}\right) \\
& =c \hbar \pi\left[\frac{n_{1}^{2}+n_{2}^{2}+n_{3}^{2}}{L^{2}}+4 \sum_{k=1}^{n} \frac{n_{k}^{2}}{L_{k}^{2}}+\frac{m^{2} c^{2}}{\hbar^{2} \pi^{2}}\right)^{1 / 2},
\end{aligned}
$$

where $m$ is the mass of the particle. The $N$-body partition function is $Z_{N}=\left(Z_{1}\right)^{N} / N$ !

The pressure $\hat{\tau}^{(k)}$ in the $k$ th direction is obtained by differentiating the free energy with respect to $L_{k}$ :

$$
\hat{\tau}^{(k)}=\frac{1}{\beta V_{(3)} V_{n}} L_{k} \frac{\partial}{\partial L_{k}}\left(\ln Z_{N}\right) .
$$

When the $L_{k}$ are small, the sum in Eq. (B1) is well approximated by the term with all $m_{k}$ being zero plus the terms with a single $m_{k}$ being one. Combining this approximation with the thermodynamic limit and replacing the sums associated with the macroscopic space by integrals, Eq. (B3) becomes

$$
\begin{aligned}
\hat{\tau}^{(k)} & =\frac{V_{(3)}}{Z_{1}^{D=3}} \frac{4 \hbar \pi c \rho}{L_{k} V_{n}} \exp \left(-\frac{2 \beta \hbar \pi c}{L_{k}}\right)\left(\prod_{i=1}^{3} \int_{0}^{\infty} d x_{i}\right) \exp \left(-\frac{\beta \hbar \pi c L_{k}}{4}\left(x_{1}^{2}+x_{2}^{2}+x_{3}^{2}\right)\right) \\
& =\frac{4 \pi \rho}{V_{n} L_{k}^{5 / 2} \beta^{3 / 2}(\hbar c)^{1 / 2}} \exp \left(-\frac{2 \beta \hbar \pi c}{L_{k}}\right)
\end{aligned}
$$

in the limit of small $L_{k}$, where

$$
\frac{Z_{1}^{D=3}}{V_{(3)}}=\left(\prod_{i=1}^{3} \int_{0}^{\infty} d x_{i}\right] \exp \left[-\beta \hbar \pi c\left[x_{1}^{2}+x_{2}^{2}+x_{3}^{2}+\frac{m^{2} c^{2}}{\hbar^{2} \pi^{2}}\right]^{1 / 2}\right]
$$


is the partition function per unit three-volume for the three-dimensional relativistic system. Equation (B5) becomes $\left(m / 2 \pi \hbar^{2} \beta\right)^{3 / 2}$ in the nonrelativistic limit of large $m$ and becomes $\left(\pi^{2}(\beta \hbar c)^{3}\right)^{-1}$ in the relativistic limit $m \rightarrow 0$.

The presure $\hat{p}$ for the macroscopic dimensions is $\hat{p}=\rho / V_{n} \beta$. For $L_{k}$ small, $\hat{\tau}^{(k)}$ is exponentially small be- cause of the factor $\exp \left(-2 \beta \hbar \pi c / L_{k}\right)$ in Eq. (B4).

For a general manifold $M^{n}$ one always finds an extremely small result because the first excitation associated with the $k$ th compactified dimension has a factor of $1 / L_{k}$ in the energy, where $L_{k}$ is the characteristic size of the $k$ th dimension.
*On leave from the City College of The City University of New York, Convent Ave. at 138th St., New York, NY 10031. Electronic address: samuelsa @iubacs.BITNET.

${ }^{1}$ T. Kaluza, Sitzunsgber. Preuss Akad. Wiss, Phys. Math. K1, 966 (1921).

${ }^{2}$ O. Klein, Z. Phys. 37, 875 (1926); Nature (London) 118, 516 (1926).

${ }^{3}$ See, for example, T. Appelquist, A. Chodos, and P. G. O. Freund, editors, Modern Kaluza-Klein Theories (Addison Wesley, Menlo Park, NJ, 1987).

${ }^{4}$ See, for example, M. J. Duff, B. E. W. Nilsson, and C. N. Pope, Phys. Rep. 130, 1 (1986), and references therein.

${ }^{5}$ See, for example, M. B. Green, J. H. Schwarz, and E. Witten, Superstring Theory (Cambridge University Press, Cambridge, England, 1987), Vols. I and II.

${ }^{6}$ V. A. Kostelecký and S. Samuel, Phys. Rev. D 39, 683 (1989).

${ }^{7}$ E. Witten, Nucl. Phys. B268, 253 (1986).

${ }^{8}$ V. A. Kostelecký and S. Samuel, Phys. Rev. Lett. 63, 224 (1989).

${ }^{9}$ C. W. Misner, K. S. Thorne, and J. A. Wheeler, Gravitation (Freeman, San Francisco, 1970).

${ }^{10}$ For simplicity, the cosmological constant is set to zero here.

${ }^{11}$ See, for example, F. W. Hehl, P. von der Heyde, G. D. Kerlick, and J. M. Nester, Rev. Mod. Phys. 48, 393 (1976), and references therein.

${ }^{12} \mathrm{~A}$ related question was considered earlier in D. G. Boulware and S. Deser, Phys. Rev. D 6, 3368 (1972).

${ }^{13}$ D. Husemoller, Fibre Bundles (Springer, New York, 1975).

${ }^{14} \mathrm{D}$. Tischler, Topology 9, 153 (1970).

${ }^{15}$ P. Candelas, G. T. Horowitz, A. Strominger, and E. Witten, Nucl. Phys. B258, 46 (1985).

${ }^{16}$ For a review of gravitational experiments, see C. M. Will, Phys. Rep. 113, 345 (1984).

${ }^{17}$ A. S. Eddington, The Mathematical Theory of Relativity (Cambridge University Press, Cambridge, England, 1922).

${ }^{18}$ H. P. Robertson, in Space Age Astronomy, edited by A. J. Deutsch and W. B. Klemperer (Academic, New York, 1962).

${ }^{19}$ I. I. Shapiro, C. C. Counselman, and R. W. King, Phys. Rev. Lett. 36, 555 (1976); see also Sec. 4.3 of Ref. 16 and references therein.

${ }^{20}$ R. D. Reasenberg, I. I. Shapiro, P. E. MacNeil, R. B. Goldstein, J. C. Breidenthal, J. P. Brenkle, D. L. Cain, T. M. Kaufman, T. A. Komarek, and A. I. Zygielbaun, Astrophys. J. 234, L219 (1979); see also Sec. 4.2 of Ref. 16 and references therein.

${ }^{21}$ E. Witten, in Shelter Island II; Proceedings of the 1983 Shelter Island Conference on Quantum Field Theory and the Fundamental Problems of Physics, Shelter Island, New York, 1983, edited by R. Jackiw, N. N. Khuri, S. Weinberg, and E. Witten (MIT Press, Cambridge, MA, 1985).

${ }^{22}$ A. Chodos and S. Detweiler, Phys. Rev. D 21, 2167 (1980).

${ }^{23}$ P. G. O. Freund, Nucl. Phys. B209, 146 (1982).
${ }^{24}$ T. Dereli and R. W. Tucker, Phys. Lett. 125B, 133 (1983).

${ }^{25}$ M. A. Rubin and B. D. Roth, Phys. Lett. 127B, 55 (1983).

${ }^{26}$ E. Alvarez and M. B. Gavela, Phys. Rev. Lett. 51, 931 (1983).

${ }^{27}$ Q. Shafi and C. Wetterich, Phys. Lett. 129B, 387 (1983).

${ }^{28}$ S. Barr and L. S. Brown, Phys. Rev. D 29, 2779 (1984).

${ }^{29}$ E. W. Kolb and R. Slansky, Phys. Lett. 135B, 378 (1984).

${ }^{30}$ S. Randjbar-Daemi, A. Salam, and J. Strathdee, Phys. Lett. 135B, 388 (1984).

${ }^{31}$ D. Sahdev, Phys. Lett. 137B, 155 (1984); Phys. Rev. D 30, 2495 (1984).

${ }^{32}$ R. B. Abbott, S. M. Barr, and S. D. Ellis, Phys. Rev. D 30, 720 (1984).

${ }^{33}$ E. W. Kolb, D. Lindley, and D. Seckel, Phys. Rev. D 30, 1205 (1984).

${ }^{34}$ C. Wetterich, Nucl. Phys. B252, 309 (1985).

${ }^{35}$ E. Kolb, Nucl. Phys. B252, 321 (1985).

${ }^{36}$ The possibility of time variation of $G_{N}$ has been examined in the context of gravitation theories in Refs. 37-43 and 22, 23, and 31 and in the context of string theories in Ref. 44. Constraints on $\dot{V}_{n} / V_{n}$ due to the time variation of the fundamental constants are considered in Refs. 26, 45-47.

${ }^{37}$ P. A. M. Dirac, Nature (London) 39, 323 (1937); Proc. R. Soc. London A165, 199 (1938).

${ }^{38}$ C. Brans and R. H. Dicke, Phys. Rev. 124, 926 (1961).

${ }^{39}$ F. Hoyle and J. V. Narlikar, Nature (London) 233, 41 (1971).

40J. D. Bekenstein, Phys. Rev. D 15, 27 (1977).

${ }^{41}$ V. Canuto, P. J. Adams, S.-H. Hseih, and E. Tsiang, Phys. Rev. D 16, 1643 (1977).

${ }^{42}$ A. Zee, Phys. Rev. Lett. 42, 417 (1979).

${ }^{43}$ A. D. Linde, Phys. Lett. 93B, 394 (1980).

${ }^{44}$ Y. S. Wu and Z. Wang, Phys. Rev. Lett. 57, 1978 (1986); Gen. Relativ. Gravit. 20, 1 (1988).

${ }^{45}$ W. J. Marciano, Phys. Rev. Lett. 52, 489 (1984).

${ }^{46}$ E. W. Kolb, M. J. Perry, and T. P. Walker, Phys. Rev. D 33, 869 (1986).

47J. D. Barrow, Phys. Rev. D 35, 1805 (1987).

${ }^{48}$ R. W. Hellings, P. J. Adams, J. D. Andersen, M. S. Keesey, E. L. Lau, E. M. Standish, V. M. Canuto, and I. Goldman, Phys. Rev. Lett. 51, 1609 (1983); see also Sec. 5.3 of Ref. 16 and references therein.

${ }^{49}$ M. Davis and J. Huchra, Astrophys. J. 254, 437 (1982).

${ }^{50}$ M. Aronson, J. Huchra, J. Mould, P. L. Schechter, and R. B. Tully, Astrophys. J. 258, 64 (1982).

${ }^{51}$ M. Davis and P. J. E. Peebles, Astrophys. J. 267, 465 (1983).

${ }^{52}$ J. Yang, M. S. Turner, G. Steigman, D. N. Schramm, and K. A. Olive, Astrophys. J. 281, 493 (1984).

${ }^{53}$ P. J. E. Peebles, Astrophys. J. 284, 439 (1984).

${ }^{54}$ E. D. Loh and E. J. Spillar, Astrophys. J. 307, L1 (1986).

${ }^{55}$ A. Sandage, Astrophys. J. 252. 553 (1982).

${ }^{56}$ A. Sandage and G. A. Tammann, Nature (London) 307, 326 (1984).

${ }^{57}$ G. Bothun, M. Aaronson, B. Schemmer, J. Huchra, and J. 
Mould, Astrophys. J. 278, 475 (1984).

${ }^{58}$ O.-G. Richter and W. K. Huchtmeier, Astron. Astrophys. 132, 253 (1984).

${ }^{59}$ E. Giraud, Astron. Astrophys. 153, 125 (1985).

${ }^{60}$ M. Aaronson, G. Bothum, J. Mould, J. Huchra, A. R. Schommer, and M. E. Cornell, Astrophys. J. 302, 536 (1986).

${ }^{61}$ M. Aaronson and J. Mould, Astrophys. J. 303, 1 (1986).

${ }^{62}$ G. de Vaucouleurs and W. L. Peters, Astrophys. J. 303, 19 (1986).

${ }^{63}$ L. Bottinelli, P. Fougue, L. Gouguenheim, G. Paturel, and P. Teerikorpi, Astron. Astrophys. 181, 1 (1987).

${ }^{64}$ A. Sandage, Astrophys. J. 331, 583 (1988).

${ }^{65}$ A. Sandage, Astrophys. J. 331, 605 (1988).

${ }^{66}$ See, for example, L. Z. Fang, T. Kiang, F. H. Cheng, and F. X. Hu, Q. J1. R. Astron. Soc. 23, 363 (1982), and references therein.

${ }^{67}$ H. R. Butcher, Nature (London) 328, 127 (1987); 330, 704 (1987).

${ }^{68}$ K. James and P. Demarque, Astrophys. J. 264, 206 (1983).

${ }^{69}$ D. A. VandenBerg, Astrophys. J. Suppl. 51, 29 (1983).

${ }^{70}$ F.-K. Thielemann, J. Metzinger, and H. V. Klapdor, Z. Phys. A 309, 301 (1983); Astron. Astrophys. 123, 162 (1983).

${ }^{71}$ R. G. Ostic, R. D. Russell, and D. H. Reynolds, Nature (Lon- don) 199,1150 (1963).

${ }^{72}$ S. Weinberg, Gravitation and Cosmology (Wiley, New York, 1972).

${ }^{73}$ Solutions describing evolving scale factors are generic. The idea that this might explain the relative sizes of spacetime and the internal dimensions was noted in Ref. 22, using a model of E. Kasner, Am. J. Math. 48, 217 (1921); see also Refs. 23, 24, 27, 30, 31, 32, and 33 .

${ }^{74}$ A. Guth, Phys. Rev. D 23, 347 (1981).

${ }^{75}$ A. D. Linde, Phys. Lett. 108B, 389 (1982).

${ }^{76}$ A. Albrecht and P. J. Steinhardt, Phys. Rev. Lett. 48, 1220 (1982).

${ }^{77}$ For sufficiently complicated models, this effect may be avoided. See, for example, Refs. 24, 27, 30, 34, and 35.

${ }^{78}$ M. Dine and N. Seiberg, Phys. Lett. 162B, 299 (1985).

${ }^{79}$ See, for example, E. H. Spanier, Algebraic Topology (McGraw-Hill, New York, 1966).

${ }^{80}$ See, for example, S. I. Goldberg, Curvature and Homology (Academic, New York, 1962), or W. Greub, S. Halperin, and R. Vanstone, Connections, Curvature, and Cohomology (Academic, New York, 1972), Vol. I.

${ }^{81}$ See, for example, M. W. Hirsch, Differential Topology (Springer, New York, 1976). 